\title{
ISA, a High Sensitivity Accelerometer in the Interplanetary Space
}

\author{
Updates after the Near-Earth Commissioning Phase of Italian \\ Spring Accelerometer - ISA
}

\author{
Francesco Santoli $^{1}$ (D) Emiliano Fiorenza ${ }^{1} \cdot$ Carlo Lefevre $^{1}$. \\ David Massimo Lucchesi ${ }^{1}$ - Marco Lucente ${ }^{1}$. Carmelo Magnafico ${ }^{1}$. \\ Alfredo Morbidini ${ }^{1} \cdot$ Roberto Peron $^{1}$ - Valerio Iafolla ${ }^{1}$
}

Received: 3 June 2020 / Accepted: 12 November 2020 / Published online: 8 December 2020

(C) The Author(s) 2020

\begin{abstract}
ISA (Italian Spring Accelerometer) is a high sensitivity accelerometer flying, as scientific payload, on-board one of the two spacecraft (the Mercury Planetary Orbiter) of BepiColombo, the first ESA mission to Mercury. The first commissioning phase (performed in the period November 2018 - August 2019) allowed to verify the functionality of the instrument itself as well as of the related data handling and archiving system. Moreover, the acceleration measurements gathered in this time frame allow to envisage the potentiality of such an instrument as a high-accuracy monitor of the spacecraft mechanical environment.
\end{abstract}

Keywords Accelerometer - BepiColombo $\cdot$ Radioscience $\cdot$ Mercury $\cdot$ Precise orbit determination $\cdot$ Non-gravitational perturbations $\cdot$ Space instrumentation $\cdot$ Structural vibrations monitoring · Gravitation

\section{Introduction}

Among the scientific objectives of the ESA mission BepiColombo (BC), an important part is given by those dedicated to the study of the Hermean geophysics, as well as to dedicated tests of the laws of gravitation, by employing the Mercury Planetary Orbiter (MPO, one of the two probes making up the mission) as a test mass in the Mercury gravity field. In order to fulfil these objectives, the Mercury Orbiter Radioscience Experiments (MORE) have been set up (Iess et al. 2020; Genova et al. 2020). The MPO spacecraft hosts a suite of instruments that enable a precise measurement of the MPO orbital motion and its dynamics, which are the key elements to reconstruct the gravitational field and rotation state of the planet and to test selected predictions of General Relativity, along with the ones of alternative theories of gravitation (Milani et al. 2001, 2002; Imperi and Iess 2015; Schettino et al. 2015, 2016; Will

The BepiColombo mission to Mercury

Edited by Johannes Benkhoff, Go Murakami and Ayako Matsuoka

F. Santoli

francesco.santoli@inaf.it

1 Istituto di Astrofisica e Planetologia Spaziali-IAPS, Istituto Nazionale di Astrofisica-INAF, Via Fosso del Cavaliere 100, 00133, Rome, Italy 
2014). In the procedure of orbit determination and parameter estimation (usually called POD - precise orbit determination), a model for the orbital dynamics of the spacecraft is fitted to the tracking data. The model is required to be sufficiently accurate to describe this dynamics at a level comparable to the information content of tracking. It has to be noticed that, while the majority of the forces acting on the spacecraft once in orbit around Mercury comes from the gravitational attraction of the primary as well as from the attraction of the other bodies in the Solar System, a non-negligible part is constituted by surface forces resultant from the interaction of the spacecraft body with particles and fields in the near-Mercury environment (the so-called non-gravitational perturbations - NGP). A precise modellisation of these forces is not easy (Lucchesi and Iafolla 2006), so that developing a NGP mathematical model with the accuracy needed for the MORE goals has been considered not viable. An effective alternative is the direct measurement of the total resulting acceleration via an onboard accelerometer. This is the solution that has been chosen for BC, employing the ISA (Italian Spring Accelerometer) instrument (Iafolla et al. 2010; Iafolla and Nozzoli 2001; Iafolla et al. 2016).

ISA is a three-axis accelerometer: it features three sensing elements arranged in a suitable geometrical configuration, each one measuring a one-dimensional component of the overall signal. These three channels can then be properly combined in order to obtain the total acceleration vector acting on a given reference point (called Vertex). The resulting time series of acceleration values can then be used as an input to the POD, allowing to remove the NGP from the list of the unknowns of the problem and hence allowing a better determination of the other unknowns (first of all the ones related to MORE scientific objectives).

After a long development and design phase by the Experimental Gravitation group at IAPS-INAF, the space-qualified version of ISA has been engineered and built by Thales Alenia Space - Italia (Milano) and integrated into MPO spacecraft. Following the successful launch on 19 October 2018 from the spaceport of Kourou (French Guiana), the instrument has been turned on for the first time on 26 November 2018. Subsequently, a first slot of tests has been successfully carried out in the Near Earth Commissioning Phase (NECP).

This work is organised as follows. In Sect. 2 the development path of ISA is briefly reviewed, starting from the pioneering work on resonant gravitational wave detectors. Section 3 is devoted to a discussion of the ISA measurements scientific objectives, in particular those linked to MORE, and it is highlighted the role of the acceleration measurements in the POD. In Sect. 4 it is shown the instrument working principle, along with details on measured signals and design choices. In Sect. 5 the instrument calibration procedures, both on ground and in flight, are discussed. Section 6 is dedicated to a presentation of the various data types produced by the instrument, along with the processing pipeline developed to prepare the scientific products. Section 7 is devoted to a first presentation and discussion of selected observations from the commissioning activities after launch.

\section{ISA, ab initio}

The history of ISA accelerometer starts at the beginning of the seventies of the past century through the studies carried out by the Roma Gravitational Waves research group (Guido Pizzella and Edoardo Amaldi, lead), devoted to the search of gravitational waves (GW) (Pizzella 2016). In those studies, one of key steps was the development of transducers able to enhance the subatomic vibrations produced by the interaction between $\mathrm{GW}$ and cryogenic gravitational antennas. The basic technology for such transducers is related to the measurement of the displacement of a sensing mass produced by a tiny force acting on 
it. The capability to sense and measure such small displacements allows to develop a wide range of instrumentation, among which ultra-sensitive accelerometers. For that reason, it is usual that people working on experimental gravitation topics often move to the research in accelerometers development.

At the beginning of nineties, the Experimental Gravitation group at CNR (National Research Council), now at INAF - IAPS (Franco Fuligni and Valerio Iafolla, lead), started an activity focused on the development of a gravitational gradiometer (Iafolla et al. 2003), composed by couples of high-sensitivity accelerometers. Afterwards, following the suggestions of late Prof. Andrea Milani (University of Pisa), the group, that at the time was led by Valerio Iafolla, started to work on the development of an accelerometer for the BepiColombo mission; to this aim, Valerio Iafolla received from ESA the instrument PI-ship. It has to be remarked that ISA is the first high-sensitivity accelerometer to be deployed in an interplanetary mission.

The accelerometer was named ISA (Italian Spring Accelerometer), as suggested from Prof. Anna Nobili (University of Pisa), to specify one of its noteworthy characteristics: the proof mass is connected to its reference through a mechanical spring. Such a peculiarity distinguishes it with respect to other approaches, such as those based on electrostatic suspension systems of the proof mass.

\section{ISA Science Goals}

In the following the ISA science goals are briefly explained and discussed, to place into context the subsequent sections. For a deeper discussion of the MORE-related goals see Milani et al. (2001, 2002), Iess et al. (2020), Genova et al. (2020). Notice that, while the bulk of goals coincides with the MORE ones, having ISA on-board BepiColombo would allow the use of its data in other areas of space science; see Sect. 3.3.

\subsection{The BepiColombo MORE Objectives}

As previously introduced, ISA main task is to contribute - via dedicated spacecraft acceleration measurements - to MORE, which foresees a set of interrelated scientific objectives and measurements based on the POD of MPO spacecraft. These measurements and objectives can be grouped into three parts, based on their main outcome. ISA contribution is part of this orbit determination procedure. Indeed, the NGP acting on the MPO in orbit around Mercury are particularly severe. The main perturbation term is given by the direct solar radiation pressure with a typical value of about $2 \times 10^{-6} \mathrm{~m} \mathrm{~s}^{-2}$, to which effects of the planet radiation (albedo and infrared emission) are added; such terms produce signals at the orbital frequency and its harmonics. The accelerations caused by all these forces acting on the MPO will be measured by ISA accelerometer. These measurements are expected to be better than analytical/numerical models of the corresponding forces (Lucchesi and Iafolla 2006).

Gravimetry Experiment This experiment is devoted to the study of the gravitational field of Mercury, including tidal effects, with the aim of characterising the planetary interior; such measurements aim to investigate the mantle structure and the crust-mantle interface (via local gravitational anomalies). The MPO can be considered a proof mass moving in the gravitational field of the planet; by determining its trajectory it is possible to derive the planet's gravity. By using a least-squares estimation procedure (where the difference between the observed orbit, derived from radio-tracking data, and the predicted orbit, computed through 
the numerical models, is minimised), the MPO orbit and the Mercury's gravitational field could be reconstructed at the same time. In particular, the static Stokes coefficients ${ }^{1}$ will be estimated up to a degree of $\sim 25-35$ (Cappuccio et al. 2020).

Rotation Experiment The purpose of this experiment is to determine the rotational state of Mercury, characterised by the polar axis direction (the inclination between the equatorial and orbital plane, also known as obliquity) and by the amplitude of the $88 \mathrm{~d}$ librations in longitude; following a method proposed by Peale $(1969,1976)$, the measurement of seconddegree gravitational harmonic coefficients, obliquity and amplitude of the libration in longitude will allow to derive some characteristics of the Mercury's core, notably the physical state of the planet core. ${ }^{2}$

The estimate of Mercury's rotational state is carried out by observing with a camera some characteristic points of the surface (landmarks); the method foresees to estimate at first the direction of the rotation axis and subsequently, after defining a reference frame rotating with constant velocity, the amplitude of the librations. To achieve this objective within the requested performance, the MPO Attitude and Orbit Control System (AOCS) needs to guarantee high precision in the reconstruction of the camera pointing, in order to obtain an accuracy of about $40 \mathrm{~m}$ in the landmarks position from the maximum orbital height; moreover, the MPO position in a Mercury-centred reference frame needs to be known with an error not greater than $3 \mathrm{~m}$. This latter condition will be obtained a-posteriori through the gravimetry experiment.

Relativity Experiment The relative closeness of Mercury to the Sun enables a series of tests of the General Theory of Relativity. ${ }^{3}$ Starting from the range measurements of MPO (through a very precise tracking from the Earth), the fundamental observable in this experiment, MORE will allow a very high accuracy determination of the Mercury-centric orbit of the MPO and of the heliocentric orbit of Mercury. This will allow carrying out an accurate test of General Relativity, determining in particular:

- Some of the parameters used to classify the metric theories of gravitation (PPN, postNewtonian parameters) (Imperi et al. 2018; Schettino et al. 2018): the $\gamma$ parameter, related to the spacetime curvature induced by a unit mass, with an accuracy of $2 \times 10^{-6}$; the $\beta$ parameter, characterising the non-linearity of gravitation, with an accuracy of $2 \times 10^{-6}$; the Nordtvedt parameter $\eta$, with an accuracy of $8 \times 10^{-6}-10^{-5}$, and the preferred frame parameters, $\alpha_{1}$ and $\alpha_{2}$, with an accuracy of $8 \times 10^{-6}$ and $10^{-6}$, respectively.

- the quadrupole moment of the Sun $\left(J_{2}\right)$ with an accuracy of $2 \times 10^{-9}-10^{-8}$;

- an upper limit for the time variation of the Newtonian gravitational constant, $(d G / d t) / G$, a parameter of interest for cosmology and galaxy formation, with an accuracy of $3 \times 10^{-13}$ per year.

\footnotetext{
${ }^{1}$ Related to a spherical harmonics expansion of the planet gravitational potential.

${ }^{2}$ The discovery by Mariner 10 of a global Mercury magnetic field spurred research on its internal physical state. Along the years, based on the results of ground-based observations and of the MESSENGER mission, evidence has been accumulated on a molten outer core (see e.g. Margot et al. 2018).

${ }^{3}$ It has to be noticed that the Mercury perihelion precession, although foreseen by the classical Newtonian calculation, presents an excess well-known from astronomical observations before the development of General Relativity by Albert Einstein. Such a difference, precisely explained by his theory, has been the first evidence that Einstein was on a good path in explaining the machinery of gravitation.
} 


\subsection{Role of Acceleration Measurements in MORE POD}

The MORE POD entails the computation of the trajectory of a physical point in the gravity field of Mercury. Usually, in this type of computation the reference point (i.e. the point the equation of motion refers to) is the centre of mass (COM) of a tracked satellite. This common approach implies that all measurements (both tracking and acceleration) are referred to the spacecraft COM. The equation of motion, in this case, can be written as:

$$
\ddot{\mathbf{r}}_{\mathrm{COM}}=\nabla U\left(\mathbf{r}_{\mathrm{COM}}\right)+\mathbf{a}_{\mathrm{NGP}}
$$

where the term $\mathbf{a}_{\mathrm{NGP}}$ represents the COM acceleration due to NGP acting on the spacecraft that should be calculated starting from ISA measurements. Because of MPO moving masses and MPO attitude motion, the acceleration sensed by ISA will be:

$$
\mathbf{a}_{\mathrm{ISA}}=-\mathbf{a}_{\mathrm{NGP}}-\mathbf{a}_{\mathrm{APP}}\left(\mathbf{r}_{\mathrm{ISA}}\right)+\mathbf{a}_{\mathrm{GG}}\left(\mathbf{r}_{\mathrm{ISA}}\right),
$$

where $\mathbf{a}_{\mathrm{APP}}\left(\mathbf{r}_{\mathrm{ISA}}\right)$ represents the apparent acceleration due to the MPO motion (around and with respect to its $\mathrm{COM}$ ) and $\mathbf{a}_{\mathrm{GG}}\left(\mathbf{r}_{\mathrm{ISA}}\right)$ represents the acceleration due to the gravity gradients between the MPO COM and the accelerometer, both depending on the instantaneous position of the masses with respect to the MPO COM. From the two equations above it results:

$$
\ddot{\mathbf{r}}_{\mathrm{COM}}=\nabla U\left(\mathbf{r}_{\mathrm{COM}}\right)-\mathbf{a}_{\mathrm{ISA}}-\mathbf{a}_{\mathrm{APP}}\left(\mathbf{r}_{\mathrm{ISA}}\right)+\mathbf{a}_{\mathrm{GG}}\left(\mathbf{r}_{\mathrm{ISA}}\right) .
$$

In order to solve Equation (3), ISA measurements should be complemented by the information on the displacement vector of ISA with respect to the moving COM, with an accuracy that would have been resulted in challenging requirements on COM stability and/or knowledge.

During BC and MORE development an alternative solution ${ }^{4}$ has been found: the POD would have been carried out by integrating the trajectory for a point fixed with respect to the MPO structure, instead of a moving COM; since the chosen point was the design position of the COM of ISA central sensor, such point has been called ISA Vertex. This choice gives MORE two main advantages:

- the COM position does not affect the parameter estimation;

- the equation of motion (3) is simplified.

Indeed, it can be easily proved (Milani and Gronchi 2010) that the equation of motion for ISA Vertex location can be written, by considering $\mathbf{r}_{\mathrm{Vertex}}=\left(\mathbf{r}_{\mathrm{COM}}+\mathbf{r}_{\mathrm{ISA}}\right)$, as:

$$
\ddot{\mathbf{r}}_{\text {Vertex }}=\nabla U\left(\mathbf{r}_{\text {Vertex }}\right)-\mathbf{a}_{\text {ISA }} \text {. }
$$

From a conceptual point of view we can say that, in its common use, the POD aims to track the entire spacecraft mass orbiting around its primary, i.e. the COM motion perturbed by the external forces. In the new approach described above the free-falling point is the vertex of ISA, whose trajectory is perturbed by all the forces acting on it, where all the forces applied to the satellite structure and transmitted through ISA spring to ISA sensing masses have to be taken into account. For what concerns the accelerometer, the main difference between the two described approaches is the distinction between what can be considered signal, i.e. the physical quantity of interest, and what can be termed noise, that affects the

\footnotetext{
${ }^{4}$ Due to Hans Reiner Schulte, see also Milani and Gronchi (2010).
} 
measurements and must be removed. In particular, the acceleration terms that in equation (3) depend on ISA motion and position with respect to the MPO COM need to be considered as noise and therefore dealt with in some way. In the second approach they are simply part of measurement and can be left into the signal used for the POD.

\subsection{Further ISA Science Objectives}

$\mathrm{BC}$ is the first deep-space mission embarking on-board a high-sensitivity accelerometer. Indeed, till now accelerometers characterised by similar performance, although different concept, have flown just in Earth orbit, in geophysics missions devoted to study the Earth gravity field: CHAMP (Reigber et al. 2006), GRACE (Flury et al. 2008), GRACE-FO (Kornfeld et al. 2019) and GOCE (Drinkwater et al. 2007). On the other hand, spacecraft typically host on-board accelerometers within the IMU (Inertial Measurement Unit), as part of the sensors helping the AOCS, although with performance not comparable to that of the previously cited missions. In this context, the expectations in terms of measurements to be carried out by an accelerometer as ISA in an environment never travelled before are really exciting and challenging.

Due to the capability of acquiring data without any access to the outer space, as needed e.g. for cameras, ISA can theoretically carry out its observations during the entire cruise phase. Considering its working principle, ISA is able to sense non-conservative forces acting on the spacecraft and perturbing its motion. However, beside signals of scientific interest (primary objective), it is sensitive as well to forces generated on-board by the spacecraft, due to its operating environment, such as mechanisms, appendages movements, sloshing, etc.; those constitute signals of interest for spacecraft monitoring (secondary objective). Obviously, the interface ISA-MPO has been designed to reduce as much as possible this noise, in order not to generate spurious signals. However, the in-cruise configuration, as Mercury Composite Spacecraft (MCS), offers a different environment to be monitored with respect to the nominal science phase at Mercury. In this frame a set of measurements potentially to be performed by ISA before the nominal science phase at Mercury has been identified, including at least the following ones:

- non-conservative forces acting on the MCS during cruise and SSC (Superior Solar Conjunction experiment);

- gravitational gradients due to planets (mainly by the monopole moment) during flybys;

- MCS accelerations during the transitions with thrusters;

- density changes within the magnetosphere (boundary regions) during flybys, depending on the experimented densities;

- detection of micrometeoroids, space debris and dust impacts.

The measurement of NGP is one of the main objectives at Mercury. However, this capability can be experimented and tuned as well during the long cruise between Earth, Venus and Mercury, when the MCS will be exposed to different solar radiation fluxes and solar wind conditions. Moreover, the journey through space regions never crossed by a highsensitivity accelerometer deserves a careful analysis of cruise data to highlight any unexpected behaviour.

Due to the location far from the MPO COM, it is expected that during the flyby phases the single sensing elements of ISA can measure as well the gravitational gradient of the planet due to the monopole component (mainly). This measurement is especially enhanced during the flybys with relatively low altitudes, such as Venus-flyby-2, in particular around the closest approach, where the contribution is higher. This aspect is particularly attractive because 
in the last years an increased interest in the use of gravitational gradiometers to measure directly the gravitational field of planets has been expressed (Griggs et al. 2014). Since in these instruments coupled accelerometers are used to detect the planet-induced gravitational gradient, such a measurement can be considered as a first on-orbit test of ISA capability as basic element for a future space gradiometer. During the cruise, about twenty-two thrusts imparted by the Solar Electric Propulsion System (SEPS) are foreseen to guarantee a safe journey to Mercury. In this frame, ISA could provide an independent measurement of the thrust and coast arc transitions in order to better assess the SEPS performance.

A further measurement to be potentially faced by ISA refers to the discontinuities encountered by the spacecraft when crossing the boundary regions of the magnetosphere. Indeed, the nine gravity assists foreseen during the cruise allow to travel through the magnetosphere of Earth, Venus and Mercury. They are characterised by boundaries resulting from the interaction between the solar wind and the planetary magnetic field, such as bow shock, magnetosheath, magnetopause, etc. The travel through these regions implies probing space volumes characterised by changes in the density of particles and ions. Potentially, changes in the spacecraft drag, especially due to charged particles (Milani et al. 1987), could be identified when flying through the magnetosphere, for instance during the crossing of the magnetopause, the outer boundary of magnetosphere. Several conditions in the flyby affect this measurement, such as the geometry, the boundary crossing time, and, especially, the encountered density changes during the flyby opportunity. Moreover, the magnetosphere shape and the particle density are deeply affected by the Sun activity. In addition, any ISA measurement needs to be correlated with complementary data from other instruments and related to magnetic field variation, plasma measurements, particles energy/density/composition.

At last, a potentiality to be investigated concerns the detection of micro-meteoroids, space debris and dust impacts on the MCS spacecraft. Indeed, the exploitation of ISA high sensitivity could establish a way to monitor small impacts on the spacecraft, if the proper conditions are met. This is especially true during the gravity assists and during the crossing of debris clouds, as for instance for comets and/or asteroid orbits.

\section{Instrument Concept and Development}

ISA accelerometer is based on quite simple basic principles, here below recalled. Nevertheless it is an high technology device, in consideration of its complexity, related to the achievement of its high sensitivity. In order to appreciate this, three parameters can be considered:

- the amplitude of the sensing masses displacement to be sensed is of the order of $10^{-12} \mathrm{~m}$;

- the maximum signal to be measured is less than a millionth of Earth gravity;

- the instrumental noise floor is about a thousandth of the seismic noise that can be recorded in the quietest on ground laboratory.

All this implies high-end solutions both for instrument design and manufacturing, as well as for its on ground testing and calibration.

\subsection{Instrument Basics}

The core of the sensor is a mechanical single-axis harmonic oscillator composed of a proof (sensing) mass connected to the accelerometer structure (hence to the spacecraft) through 
Fig. 1 Sketch of a sensing element including the pick-up and actuation plates
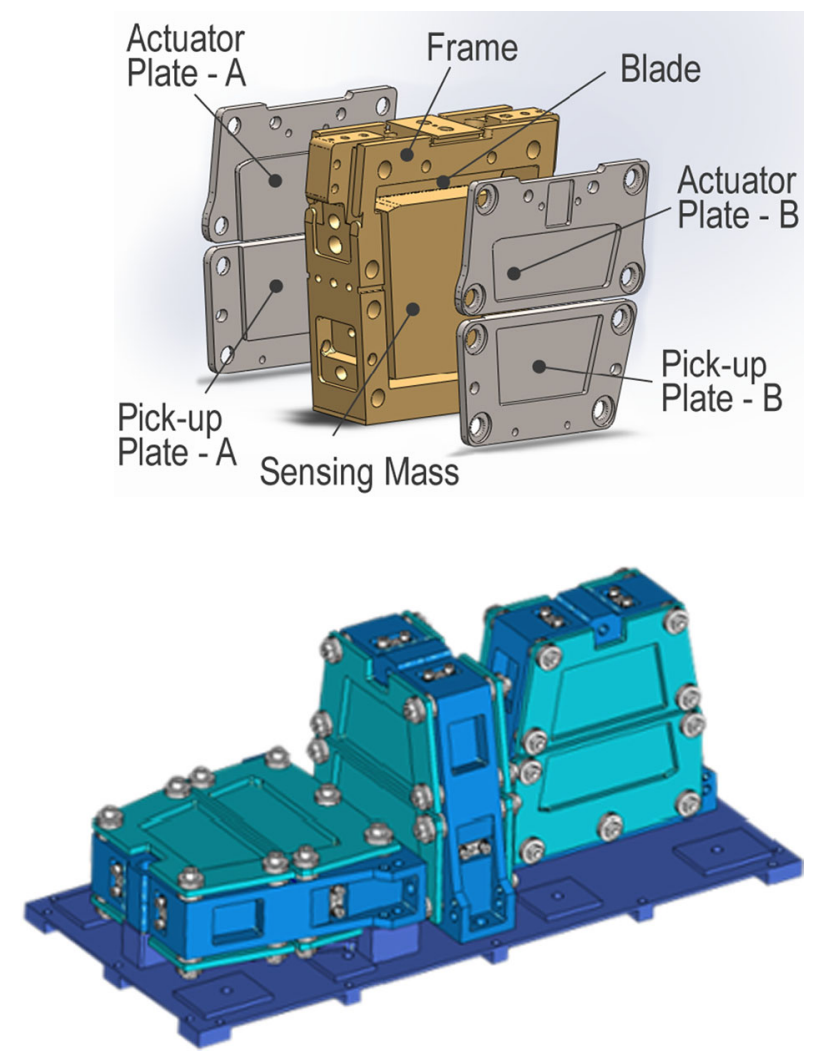

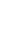

Fig. 2 Arrangement of sensing elements 
Fig. 3 IDA exploded drawing showing its various parts

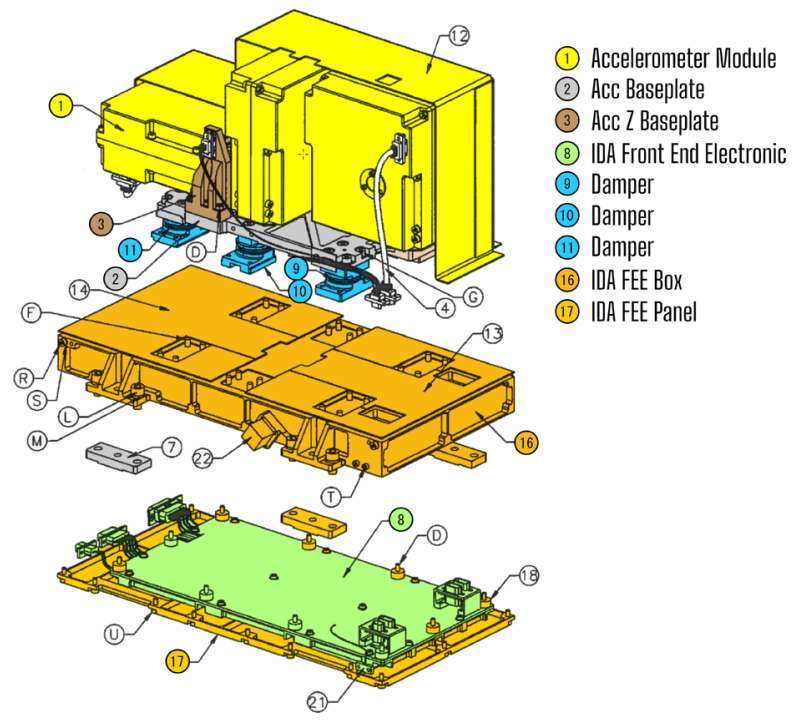

accelerometers supporting structure. The FEE hosts the analog-to-digital electronics, necessary for the pick-up of acceleration signals, and the digital-to-analog conversion for the actuators voltage supply. The FEE is responsible also for Active Thermal Control (ATC), thermometers reading and heaters driving. IDA communicates with a second unit, the Instrument Control Electronics (ICE), responsible for the digital control of the entire instrument and for the telecommand/telemetry (TC/TM) communication with the spacecraft, using the SpaceWire protocol.

\subsection{Acceleration Measurement Definition and Expected Performance}

Although the fundamental accelerometer measurement concept appears to be rather simple, it is necessary to define it precisely, in order to proper integrate the acceleration measurements in the POD procedure as outlined above. Furthermore, it is necessary to precisely characterise the instrument performance. This issues are discussed in the following.

Measured Signals and Instrument Reference Point ISA measures the acceleration vector of its Vertex point, as discussed in Sect. 3.2 concerning the alternative RSE approach. Such acceleration results from the combination of:

- NGP acting on the spacecraft, the main one being the direct solar radiation pressure;

- inertial accelerations due to spacecraft rotations around its COM and movements of COM itself with respect to spacecraft body;

- gravity gradients between ISA Vertex and spacecraft COM.

Since the three ISA sensing elements measure the acceleration components acting at the three positions of the three COM of the respective sensing masses, the acceleration at Vertex is computed applying corrective factors to compensate for the different inertial and gravity gradient accelerations between the actual measurement points and ISA Vertex. Such corrective terms are computed relying on the attitude data provided by the spacecraft AOCS.

In principle, any point of the spacecraft body-fixed reference frame could have been chosen as reference point for the POD procedure; in order to reduce the errors related to 
the computation of the above-mentioned corrective terms (to take into account the different positions of the three sensing masses with respect to the chosen point), ISA Vertex has been selected to be the design position of the COM of the central ISA sensing mass.

Measurements Reference Frame The ISA Vertex acceleration, above defined, is expressed as its components along the axes of a reference frame fixed with respect to ISA (and spacecraft) structure. Such a reference frame has been defined, according to the sensing axes alignment calibration described in Sect. 5.3, in such a way to minimise the angular separation between its axes and the actual direction of the respective sensing axes.

Measurements Frequency Band and Expected Amplitude ISA will measure the variations of acceleration within the frequency band $3 \times 10^{-5} \mathrm{~Hz}-10^{-1} \mathrm{~Hz}$. A data sampling frequency of $10 \mathrm{~Hz}$ has been set.

During the BepiColombo Mercury Science Phase, the overall peak-to-peak amplitude of the signal harmonics within such a frequency band is expected not to exceed the level of $3 \times 10^{-6} \mathrm{~m} \mathrm{~s}^{-2}$. It has to be noticed that the overall amplitude of the acceleration signal, including the harmonic content below $3 \times 10^{-5} \mathrm{~Hz}$, down to DC (hence not measured), is of about $3 \times 10^{-5} \mathrm{~m} \mathrm{~s}^{-2}$.

Measurement Error Requirements on the ISA measurement error (named "Total measurement error") has been defined considering two different quantities, specifically defined:

"Measurement deterministic error" , the part of the Total measurement error formed by the harmonic components in the MPO orbital period that are in the ISA measurement frequency band;

"Measurement total noise" , the difference between the Total measurement error and the Measurement deterministic error.

For what concerns the Measurement deterministic error, the needed performance level is defined such that the maximum amplitude $(0.5 \times$ (Max - Min $)$ over one orbit $)$ of the "Measurement deterministic error" affecting each component of ISA measurements must be below the value of $10^{-8} \mathrm{~m} \mathrm{~s}^{-2}$. The actual performance during Mercury Science Phase will depend mainly on the spacecraft attitude behaviour; during nadir pointing phases, the expected value is of about $3 \times 10^{-9} \mathrm{~m} \mathrm{~s}^{-2}$.

The required performance level for the Measurement total noise is defined as a profile of normalised acceleration power spectral density. The Measurements total noise affecting each component of ISA measurements must be below the value indicated in Fig. 4 (black line). The expected performance during Mercury Science Phase, when the spacecraft will be nadir pointed, is shown in the same Figure (red line). Such a performance level has been demonstrated to guarantee the fulfilment of ISA and MORE science goals. All the above ISA measurements characteristics have been set during the development and definition of MORE procedures and represent the top-level source of ISA instrument requirements (Iafolla et al. 2007).

\section{Instrument Calibration}

The instrument calibration is a prerequisite in order to fully capitalise on its capabilities within the frame of MORE. Indeed, the end-to-end measurements performance depends on 
Fig. 4 Random noise performance: expected (red line) and required (black dashed line)

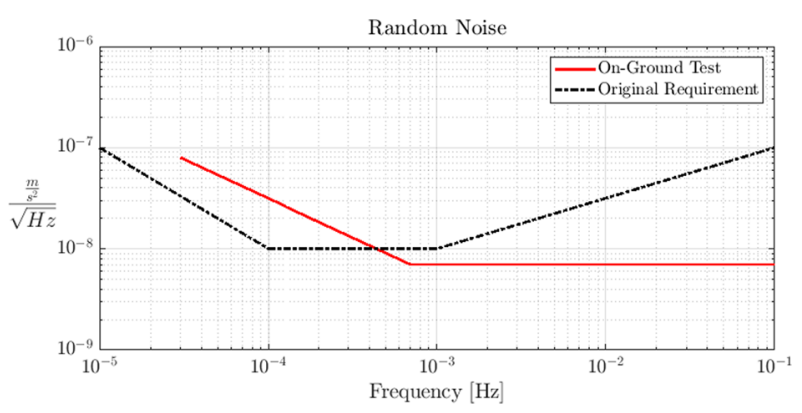

different elements: the instrument intrinsic performance (such as noise, etc.), the characteristics of the environment in which the instrument operates (such as thermal environment, mechanical vibrations, etc.) and, last but not least, the availability of suitable calibration data.

In this context, as calibration data it has to be meant the ensemble of all instrument parameter values needed to derive scientifically valuable data, starting from instrument raw measurements. Three main calibration types have been defined:

- calibration of the transduction factors;

- calibrations of actuators;

- calibration of the sensing axes alignments.

Two of these, namely those related to actuators and sensing axes alignments, have been performed on ground. The first one, although was determined on ground as well, has to be carried out necessarily in orbit, as part of the nominal operations, since the transduction factor of each sensing element depends on the setting of several parameters not predictable in advance.

Moreover, in addition to the different calibrations, a further characterisation of the sensors was carried out, the zero- $g$ measurement, i.e. the measurement of the sensing mass rest position when the sensor is in free-fall conditions (named briefly zero- $g$ ), condition that would be observed in orbit. Indeed, the sensor offers the best performance when the sensing mass operates around this equilibrium position. Ideally, each sensing element should have the proof mass centred with respect to the plates (mechanical centre); however, the mechanical manufacturing introduces unavoidable stresses in the structure (asymmetries and non-uniformities into the material and in the thin spring). Consequently, the sensing masses will result off of their design position, typically by $20 \mu \mathrm{m}-100 \mu \mathrm{m}$. Therefore this position needs to be identified and corrected to guarantee the requirements. ${ }^{6}$ ISA Science Team at IAPS-INAF developed an experimental procedure and a set-up to measure the zero- $g$ position, carrying out a dedicated measurement campaign. More details can be found in Fiorenza et al. (2016).

\subsection{Calibration of the Transduction Factor}

This calibration constitutes the basic characterisation for each sensor since it correlates the amplitude of output signal to that of the acceleration acting on the sensor. As previously said, an acceleration input on a single sensor induces a displacement of the central proof mass, which in turn causes a bridge unbalance and a modulation of the bias voltage; this signal is

\footnotetext{
${ }^{6}$ On ground, gravity modifies this displacement, making very difficult its measurement.
} 
hence digitised and demodulated, to obtain the raw measurement signal. The transduction factor is the proportionality term between the physical acceleration signal and the electrical output provided by each sensing element. However, its value is a function of several instrument parameters, such as gain of amplification chain, proof mass equilibrium position, operating temperature, applied damping factor, and so on. For this reason the transduction factor needs to be calibrated often in flight and this constitutes a routine operation to be carried out before any measurement phase. The ISA internal actuators are used to this aim by applying a reference acceleration to the proof mass and measuring the corresponding output voltage. The actuators have a precision of $600 \mathrm{ppm}$ and an absolute accuracy of $1 \%$ (Pisani et al. 2015, 2016). It has to be noticed that an estimate of the measurements scale factor (on time intervals of the order of Mercury orbital period) is foreseen in the POD (Cappuccio et al. 2020): this should allow an improvement on transduction factor knowledge.

\subsection{Calibration of Actuators}

Actuators are a fundamental element of each accelerometer sensing element, indeed by applying to its plates proper voltage signals they produce an electrostatic force on the proof mass. The force is applied to set the working point of the proof mass (centering), to damp proof-mass oscillations, to tune the sensitivity by adjusting the effective spring constant and, last but not least, to provide a known force signal that can be used to calibrate the transduction factor. The latter capability will be used in flight whenever any of the instrument parameters affecting the transduction factor changes. How often it shall be needed will be defined during instrument commissioning in Mercury orbit; a preliminary estimate is of once every 1 to 30 days. The calibration of actuators allowed to find the relationship between the voltage signal applied to the actuator plates and the force (and equivalent acceleration) applied to the sensing mass.

Taking into account the structure of the sensor as depicted in Sect. 4, the actuators calibration procedure was based on two steps: first, the pick-up plates were calibrated to find the relationship between sensor output and acceleration applied, then the actuators were calibrated comparing the voltage unbalance applied to the actuators to the sensor output provided by the pick-up plates. At the end of the process, the relationship linking the acceleration applied through the actuators and the voltage was obtained.

This calibration of actuators was carried out during a long measurement campaign in June-July 2015 by IAPS ISA Science Team at INRIM (Italian Institute for the Metrological Research) premises in Torino, with the support of Thales Alenia Space-Italia and INRIM itself. The measurements allowed to determine the calibration curve with an accuracy of 600 ppm. More details on this calibration can be found in Pisani et al. $(2015,2016)$.

\subsection{Calibration of Sensing Axes Alignments}

The objective of this calibration is to measure the directions of the three sensing axes, i.e. the sensing direction of each element, with respect to a properly defined instrument reference frame. Since ISA measures the projection of the acceleration on the sensing axes, through the knowledge of such directions and taking into account the actual sensing masses positions (as explained in Sect. 4.2), it is possible to reconstruct the acceleration vector.

The ISA Science Team at IAPS developed the calibration procedure and the experimental set-up aimed at identifying, on ground, the direction of ISA sensing axes with respect to an optical reference frame mounted on the IDA unit. A $35^{\circ}$-tilted optical cube fixed to the ISA 
Fig. 5 ISA flight model along with UOAF and COC optical cubes
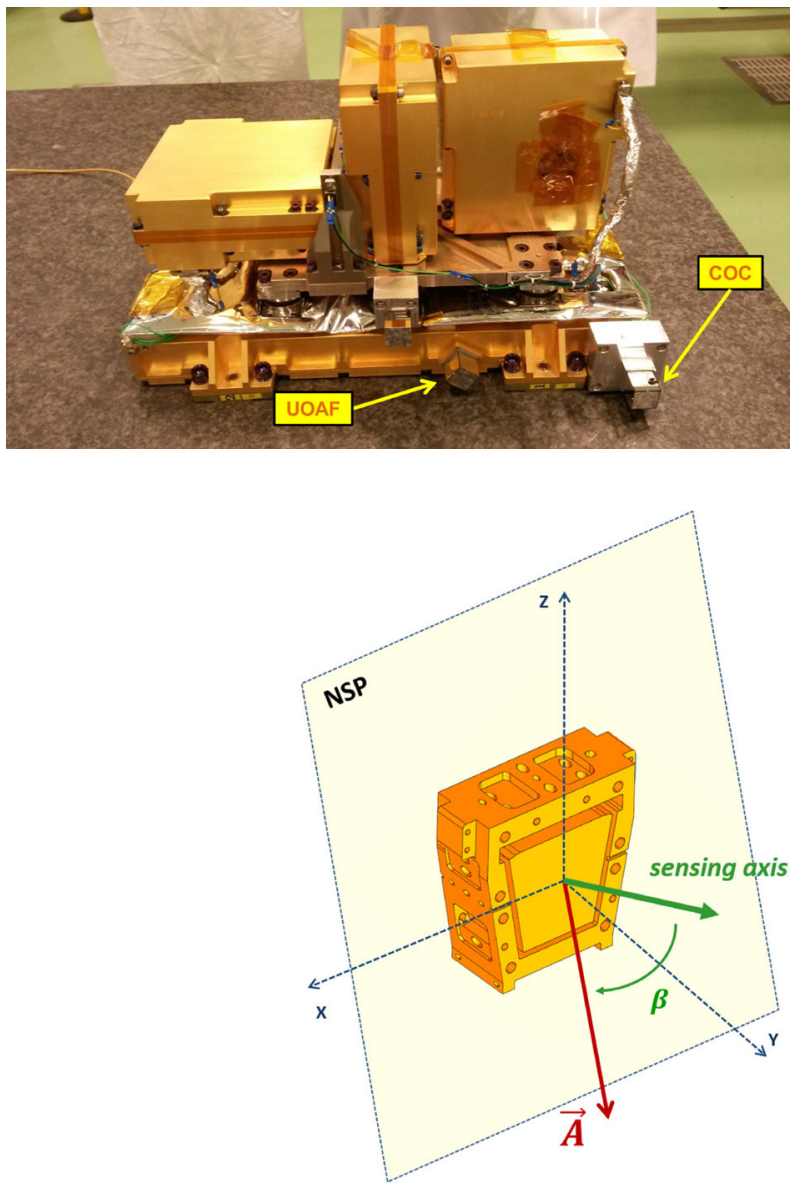

Fig. 6 Rotation of the sensor (hence of the sensing axis) by an angle $\beta$ with respect to an applied acceleration $\mathbf{A}$

basement, named UOAF (Unit Optical Alignment Frame) and used for the instrument alignment with respect to the spacecraft, was available to be used as reference. However, a second optical cube (COC, Calibration Optical Cube) was added to simplify the experimental set-up and the related procedure. Figure 5 shows the ISA flight model and the UOAF/COC optical cubes.

The calibration foresees basically 1) the excitation of each sensing element by applying inertial accelerations through a controlled linear stage, 2) the measurement of acceleration directions with respect to the COC, through optical displacement sensors, and 3) the correlation of these measurements with the instrument output to determine the direction of the sensing axes.

The sensing axis direction (SA) is measured through the evaluation of the accelerometer response, $R$, when it is rotated with respect to an applied acceleration, A. Referring to Fig. 6, the sensor response is solicited by the component of acceleration A projected along the sensing axis, i.e. A.SA. For accelerations applied in any direction perpendicular to the sensing axis, the sensor response would be zero; all the accelerations satisfying such a condition identify a plane, named Non-Sensitivity Plane (NSP).

It is worth noticing that the response change (with respect to the rotation angle) undergoes larger variations, hence increased resolution, when the rotation is performed around the NSP. 
Fig. 7 Scheme of the NSP, identified by $\mathbf{V}_{\mathbf{1}}$ and $\mathbf{V}_{\mathbf{1}}$, and of the sensing axis (e.g., $X$ ) with respect to the COC frame. $\Delta \mathbf{x}$ is the applied solicitation

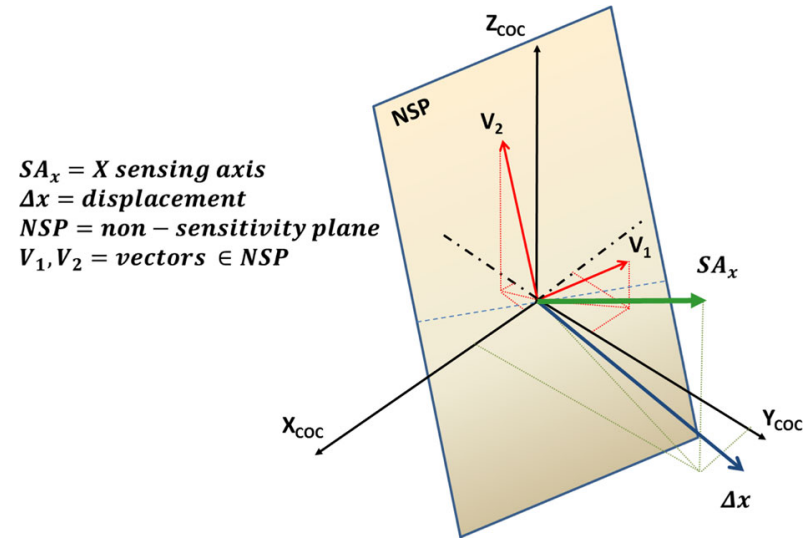

For this reason, the measurement of the sensing axis direction is led back to the identification of the NSP. In the calibration procedure the NSP is obtained by identifying two non-parallel vectors belonging to it, $\mathbf{V}_{\mathbf{1}}$ and $\mathbf{V}_{\mathbf{2}}$ (see Fig. 7). Then the sensing axis direction, being the perpendicular to it, is derived straightforwardly.

The measurement of the $\mathbf{V}_{\mathbf{1}}$ and $\mathbf{V}_{\mathbf{2}}$ vectors is carried out in two phases. In the first phase a linear stage forces ISA to move and provides a periodic inertial acceleration. Meanwhile the sensor is progressively rotated till to null its response; in this condition the acceleration direction belongs to the NSP and identifies the first displacement vector $\mathbf{V}_{\mathbf{1}}$. Three triangulation laser displacement sensors aimed at the COC faces measure the projections of this vector on the COC frame. The second phase is a repetition of the first one on the same sensor, rotated of about $90^{\circ}$ around the sensing axis. In such a way a second displacement vector, $\mathbf{V}_{\mathbf{2}}$, lying on the NSP, is identified.

Repetition of these measurements allows to collect several $\mathbf{V}_{\mathbf{1}}$ and $\mathbf{V}_{\mathbf{2}}$ vectors. The best plane fitting the experimental data is derived by applying a least-squares fit to the measured displacement vectors; this way the NSP is determined. Finally, once the plane has been estimated, the sensing axis is directly obtained as the perpendicular to it.

An experimental set-up was designed and assembled with the aim of performing the above-described calibration procedure. The assembly was made up by taking into account the position to be arranged by the sensors to identify the sensing axes direction, resulting into six configurations, equivalent to two sensor positions for each axis (pendulum and flag arrangement). The main elements of the calibration facility are shown in Fig. 8. The accelerometer is secured inside a mechanical interface box, to make easier the mounting and management of the instrument during the measurements.

The mechanical interface box allows to move the IDA unit in the different positions with respect to the linear stage and to the local gravity, needed to identify the two displacement vectors for each of the three sensors. The COC cube is fixed close to a ISA corner. A rotation stage, placed under the mechanical box, allows to rotate the accelerometer around a vertical axis. Below it, a high-precision linear stage is used to apply selected accelerations to ISA. All the assembly is fixed to an aluminium levelling plane, used to align the sensing axis in the horizontal plane, so that the gravity component along the sensing axis does not exceed the instrument dynamics. At last, all the system leans against a ground-based massive optical bench, providing a very stable, planar and stiff basement. A triplet of orthogonal lasers, placed (during measurements) close to the COC cube, allowed to monitor the relative displacement of each sensor with respect to it. 
Fig. 8 Details of the calibration facility mounted on the optical bench in clean room during the ISA calibration: 1) ISA FM, 2) COC, 3) mechanical box, 4) rotation stage, 5) linear stage, 6) levelling plane, 7) laser, 8) optical bench, 9) micrometric screws

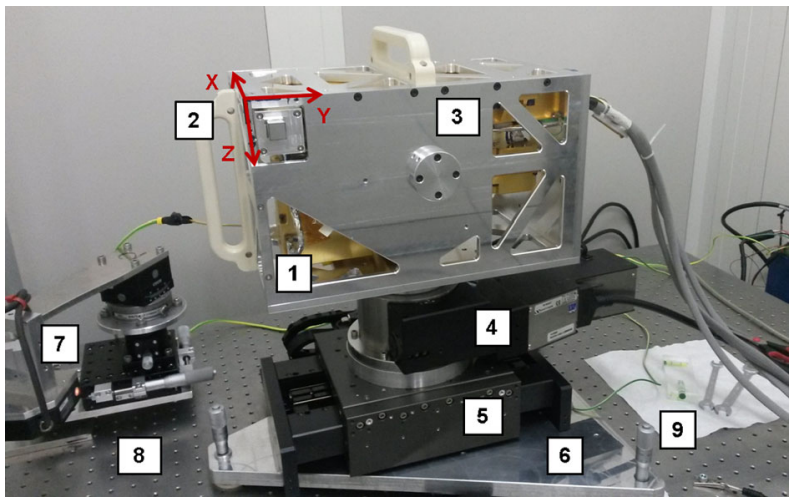

This calibration of ISA FM was carried out in November 2015, allowing to reach an accuracy in the direction measurement within $20^{\prime \prime}-30^{\prime \prime}$ per axis (at $2 \sigma$ ), well below the $100^{\prime \prime}$ required to allow the fulfilment of requirement on Measurement deterministic error (see Sect. 4.2). It has to be noticed that the three sensing axes are not exactly orthogonal to each other (due to unavoidable manufacturing tolerances). In order to ease data exchange, the reference frame representative of the ISA sensing axes is identified with the Instrument Line of Sight (ILS) reference frame. It is defined as the orthogonal frame with origin into the ISA Vertex (coincident with the COM of Y sensing mass) and such to minimise the angular separation with respect to the measured ISA sensing axes (see also Sect. 4.2).

\section{Scientific Data Processing}

While the instrument testing, calibration and operation planning activities were being carried out, an ISA scientific data processing and archiving architecture has been developed in cooperation between ISA science team and ESA BC Science Ground Segment (SGS). The ISA data processing pipeline is designed to run on SGS servers and, as per the existing scientific data exploitation agreement between ISA PI and ESA, it will provide validated data, fully compatible with ESA data archiving and quick-look analysis systems (Koschny et al. 2010; Perez-Lopez et al. 2016).

\subsection{Data Products}

The data produced by the instrument, once processed by the pipeline, are archived according to the ESA standards (ESA 2020a), which make use of Planetary Data System version 4 (PDS4) (NASA 2020). Not all the TM produced by ISA will be long-term archived. The data that will be archived are the ones necessary to build the scientific products, including calibration data and all the related housekeeping. To this set ancillary information is added, from both on ground calibration activities and spacecraft TM. All of this data have been identified, grouped and assigned a proper Processing Level ID (PDS4 Value).

The data have been organised according to the processing levels shown in Table 1. Level 1 data are bare TM files, as produced by the instrument, time-tagged and sent to the ground. Level 2 and 3 data contain the same information, i.e. the one necessary as input to the processing pipeline; at level 3 , this information has been reformatted in order to be directly usable by the pipeline. Level 4 data contain the output of the pipeline, in which each sensing 
Table 1 ISA processing levels, classified following both internal and PDS4 conventions

\begin{tabular}{lll}
\hline Level & Internal & PDS4 \\
\hline 1 & TM-PACKETS & Telemetry \\
2 & RAW-PRODUCTS, SUPPLEMENTARY & Raw \\
3 & PRE-PROCESSED & Raw \\
4 & REDUCED & Calibrated \\
5 & POST-PROCESSED & Derived \\
\hline
\end{tabular}

element time series has been properly calibrated and reduced to the Vertex; as such, they can be considered the 'official' products. Level 5 data introduce further processing steps (e.g. filtering), dedicated to particular applications. It has to be noticed that at this level several different datasets (with e.g. varying filtering) can coexist.

\subsection{ISA Processing Pipeline Description}

The ISA processing pipeline is the ensemble of software developed to process the ISA TM products (PDS4 Telemetry) to deliver science-valuable products (PDS4 Derived). A single ISA observation is defined as a series of consecutive acceleration measurements ${ }^{7}$ long enough to cover the acceleration evolution in a physically representative and meaningful time span. Indeed, since ISA is a relative accelerometer, each single acceleration measurement is meaningless if not related with the others. This definition impacts the way the instrument is operated and the data are handled and archived.

ISA Processing Pipeline Architecture The working scheme of ISA processing pipeline is shown in Fig. 9. ISA TM data are produced according to the SCOS-2000 standard (ESA 2020b). Once received on ground, the TM packets are ingested by ESA dedicated facilities and archived as Telemetry processing level in BC Data Archive. ISA pipeline starts by unpacking this TM. The TM2RAW processing step extracts parameters who need further processing from the TM and takes trace of any event that can cause inhomogeneities in the data (i.e. instrument mode changes, temperature control changes, MPO manoeuvres, attitude mode change). At the end of this processing step homogeneous RAW data blocks are produced and archived.

Data Pre-Processing ISA basically produces an uninterrupted time series of data; in order to be correctly handled it is necessary to divide the data in homogeneous blocks that can be processed using the same parameters and routines. The final output (i.e. PDS4 Derived Products) will constitute a set of measurement sessions with variable length. It has to be noticed that, since the measurements are band limited (see Sect. 4.2), it is not possible to simply connect two consecutive sessions side-by-side to obtain a single one. Indeed measurements of each session are taken with a different (arbitrary) zero or mean value; moreover, connecting sections would not provide additional information on the harmonic content below the band lower limit of $3 \times 10^{-5} \mathrm{~Hz}$, since instrument stability for frequencies lower than that is not guaranteed.

In order to carry out such an approach, pre-processed data will be divided into homogeneous blocks obtained dividing the data time series, at first, according to the instrument

\footnotetext{
${ }^{7}$ We recall that ISA samples the three acceleration observables every $0.1 \mathrm{~s}(10 \mathrm{~Hz}$ sampling rate).
} 


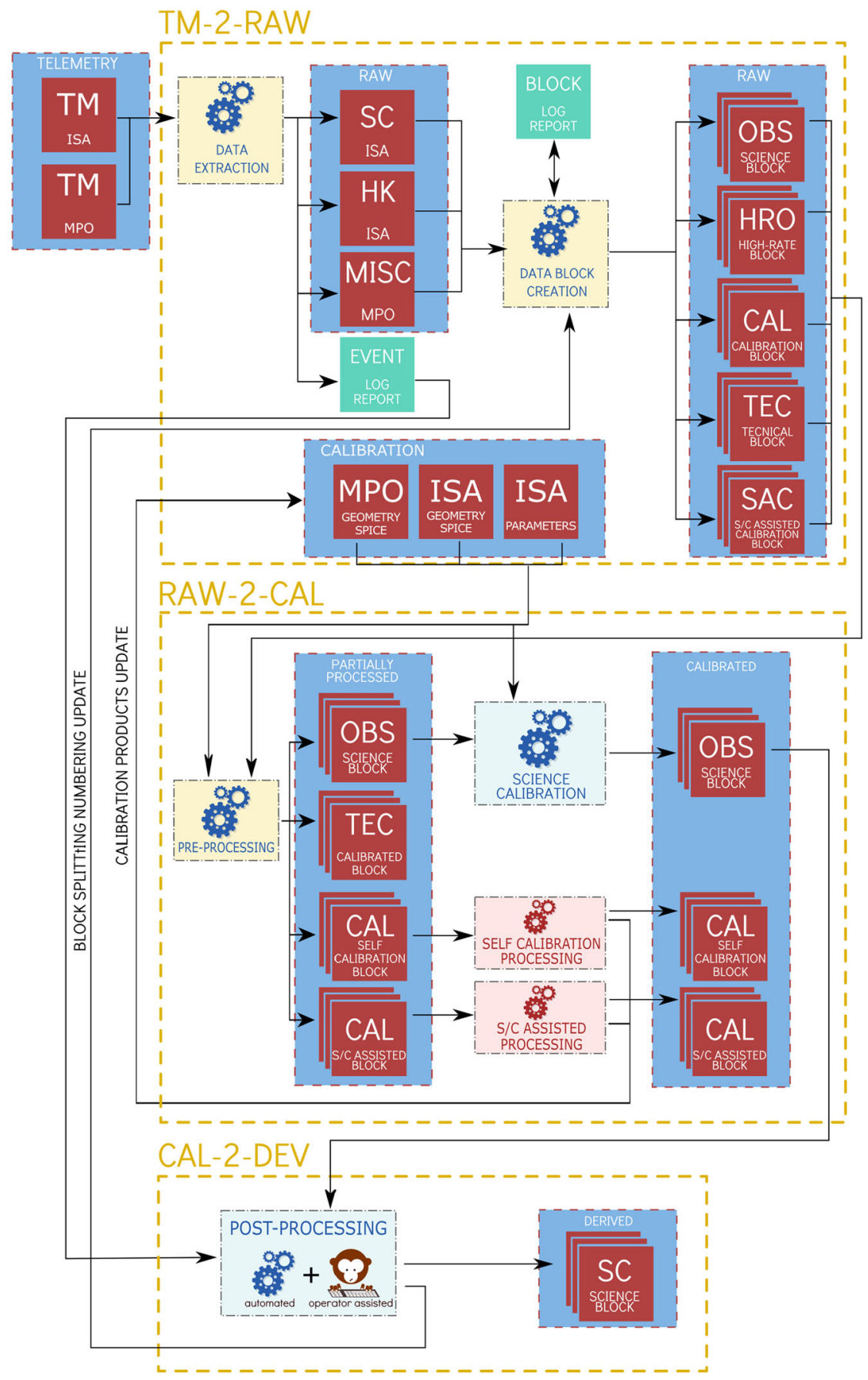

Fig. 9 ISA processing pipeline flowchart 
Table 2 ISA data block types and IDs as mapped into operative modes

\begin{tabular}{lll}
\hline ISA operative mode & Block type description & Block type ID \\
\hline Boot & Technical mode & TEC \\
Stand-by & Technical mode & TEC \\
Safe & Technical mode & TEC \\
Configuration & Technical mode & TEC \\
Diagnostic & Technical mode & TEC \\
Self Calibration & Calibration & CAL \\
Chain Calibration & Calibration & CAL \\
Scientific Observation & Nominal science observation & OBS \\
High-rate Observation & High-rate observation & HRO \\
SC Assisted Calibration & Calibration based on spacecraft movements & SAC \\
\hline
\end{tabular}

operative mode in which they are produced (following the scheme in Table 2), and then further dividing it, in order to have blocks characterised by the same instrumental setting and environmental conditions.

Data Calibration and Reduction Pre-processed data are then ingested by RAW2CAL processing step. The acceleration measurements are at first calibrated by applying the scale factors, resulting from a dedicated branch of the pipeline; then the measurements are processed to reconstruct the acceleration vector at ISA Vertex, as described in Sect. 4.2.

The diagram reported in Fig. 10 describes the science data reduction procedure. It takes as input the following data:

- calibrated acceleration measurements by the three ISA sensors;

- spacecraft attitude measurements by the AOCS;

- position and attitude of the spacecraft around Mercury (or other primary) by SPICE kernels (JPL-NASA 2020; Costa 2018);

- geometrical information from on ground ISA and spacecraft calibrations.

Usually, the considered AOCS measurements are angular velocity and angular acceleration coming from the AOCS Kalman filter estimation procedure. They are here used to calculate the apparent acceleration at the three ISA sensors COM. Attitude and position with respect to Mercury (or any other primary) are used to compute the gravity gradients between the $\mathrm{X}$ and $\mathrm{Z}$ sensors and the Y COM (i.e. the ISA Vertex). All these acceleration corrections will be computed with an accuracy compatible with ISA measurements expected performance.

Data Post-Processing Calibrated scientific products are injected into the CAL2DEV processing step, producing, as output, scientific DERIVED products. Post-processing is intended to prepare the data for their use within MORE and in general for their scientific exploitation. This step, differently from the previous ones, will not be completely automated, but the intervention of an operator will be needed to ensure data homogeneity and quality.

\subsection{Graphical Data Visualisation}

Data representation is crucial for a rapid and correct understanding of what an instrument is measuring and if it is performing as expected. The simplest visualisation of ISA data is 


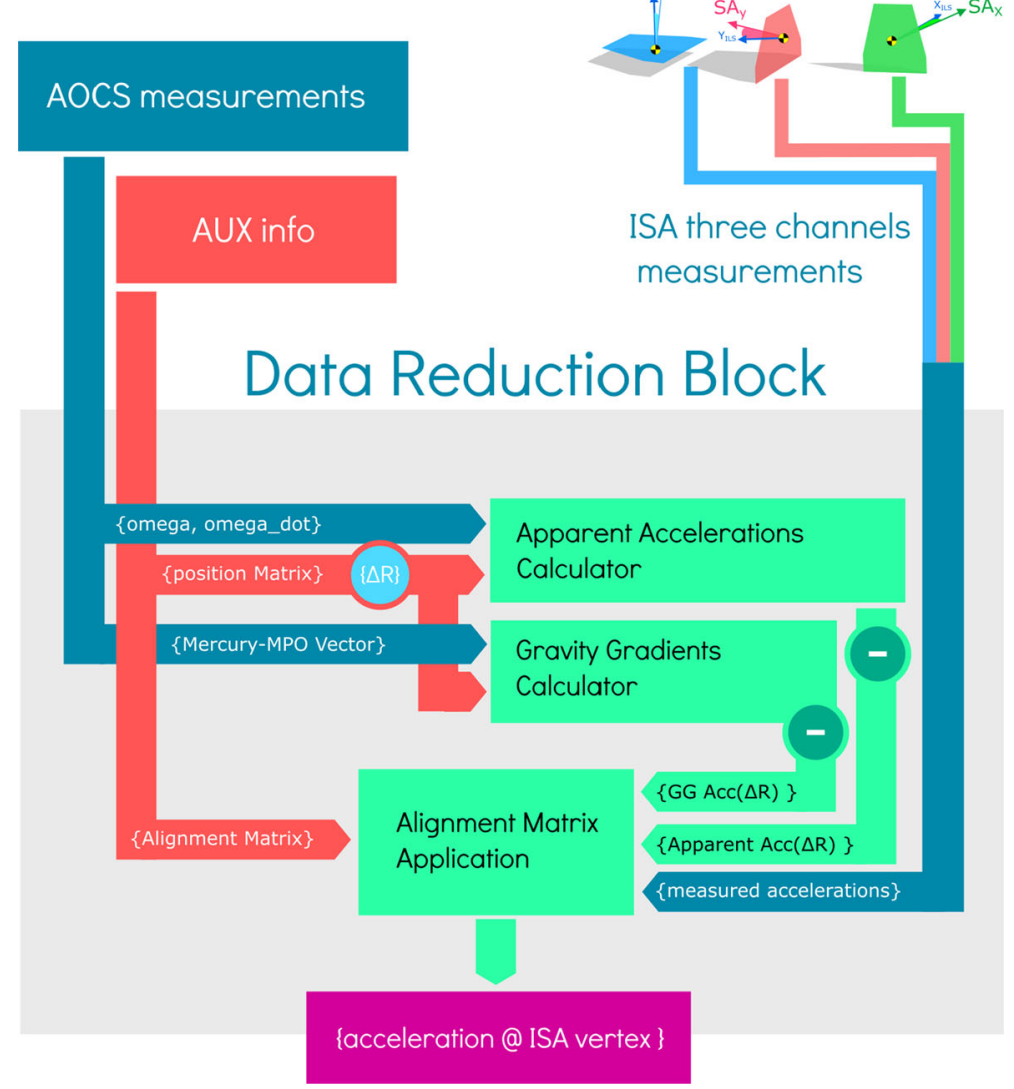

Fig. 10 ISA data reduction diagram

the time plot of the three components of the acceleration vector (in case of reduced data) or of the signal measured by the three channels (in case of raw data). This kind of visualisation gives the user a first immediate glimpse of the signal overall amplitude and allows to compare the behaviour of the three channels/components as shown in Fig. 11.

Another common visualisation of band-limited time series is provided by their spectrum; such a visualisation is particularly useful for accelerometer data which, by their nature, usually have a well-defined frequency signature. Indeed, accelerometer data analysis is focused on the separation of scientifically valuable signals from stochastic noise or deterministic disturbances due to on-board mechanisms. By a proper selection of parameters (e.g. windowing and normalisation factor), it is possible to put in evidence different signal features.

Figures 12 and 13 show two power spectral density (PSD) plots of the same acceleration signal; the first one (produced using Blackman windowing) allows to evaluate the amplitude of the signal peaks; the latter, instead, is a normalised PSD and allows to evaluate the level of the random noise, such as the measurements noise floor. In both plots it is possible to see the mechanical oscillator resonance frequency, at about $3.5 \mathrm{~Hz}$.

Moreover, when approaching to a long time series of measurements, the user can be interested to the changes of the signal spectrum content over the time. To fulfil this need a spectrogram visualisation could be used. A spectrogram is, basically, a PSD repeated over 


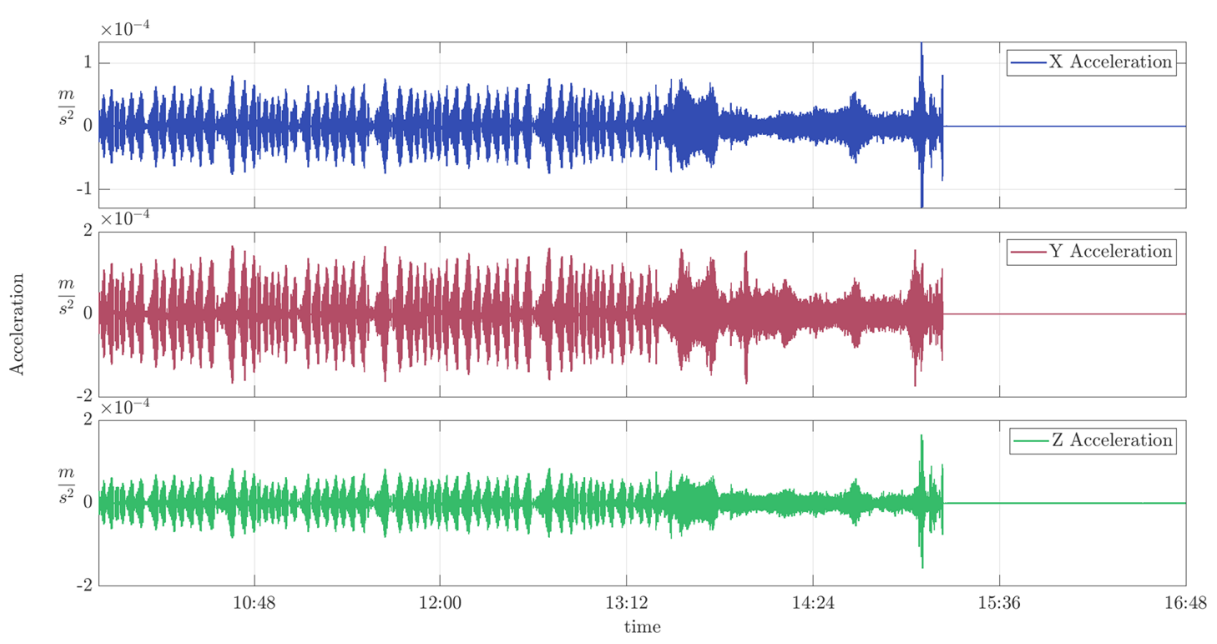

Fig. 11 Time series of ISA acceleration measurements, taken on 19 August 2019, from 10:25:00 to 18:25:00

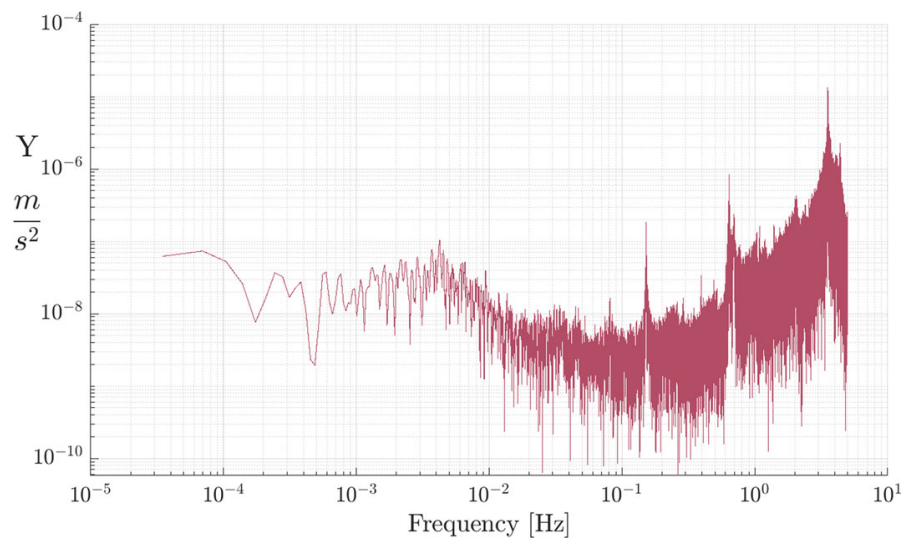

Fig. 12 ISA Blackman PSD for the accelerometer 1 (ISA Y) on 19 August 2019, from 10:25:00 to 18:25:00

contiguous and partially overlapped time spans. This kind of representation can quickly show changes in the signal spectrum, highlighting in particular how peaks evolve during the considered time span. An example of how this kind of analysis can highlight such an effect is reported in the spectrogram of Fig. 14. The bell-shaped patterns represent the change of amplitude and frequency of the vibrations induced by the spacecraft reaction wheels, whose speed was varying during a slew manoeuvre. This is an effect totally hidden in other data representations such as the ones in Figs. 11, 12 and 13.

\section{First Commissioning Outcomes}

About one month after the launch (on 19 October 2018, local time), BC spacecraft and payloads entered into the Near-Earth Commissioning Phase (NECP). This is a period devoted to perform dedicated tests to verify the health of spacecraft science instruments, its propulsion 


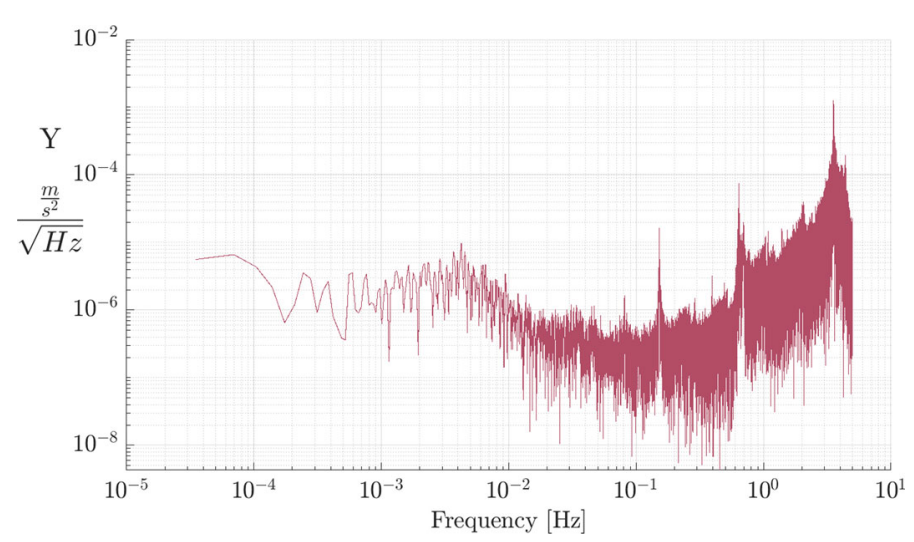

Fig. 13 ISA Normalised PSD for accelerometer 1 (ISA Y) on 19 August 2019, from 10:25:00 to 18:25:00

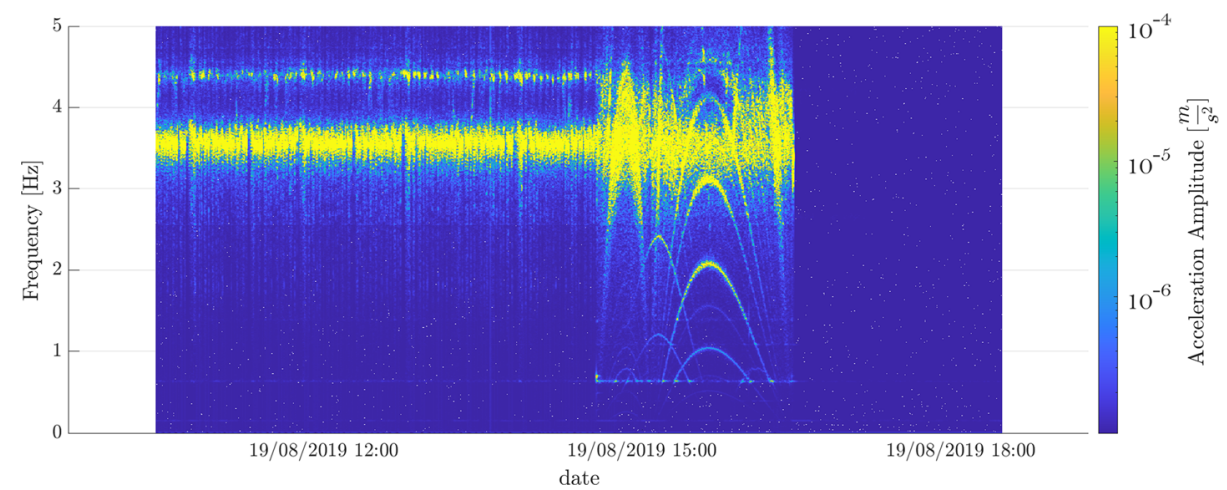

Fig. 14 ISA spectrogram for accelerometer 1 (ISA Y) on 19 August 2019, from 10:25:00 to 18:25:00. Note the signatures of the four reaction wheels from 14:35:00 to 16:27:00

system and other platform systems. ISA commissioning activities started on 26 November 2018 at 13:26:48 UTC with the first switch-on. In the following are described the main outcomes of the activities performed in two NECP slots: the first one from the switch-on to 15 December 2018; the second one from 26 to 28 August 2019.

\subsection{ISA Functional Verification}

Preliminary tests, successfully passed, were aimed at verifying the integrity and operation of the instrument after the launch vibration and shock. A series of health and functionality checks were carried out, including:

- tests of all instrument operative modes and main software functions, including fault detection, isolation and recovery (FDIR) functionalities;

- functional verification of ISA thermal control system;

- verification of the correct thermalisation of sensors;

- measurement of sensing masses equilibrium position (with actuators switched-off), for the first time in zero- $g$ conditions; 


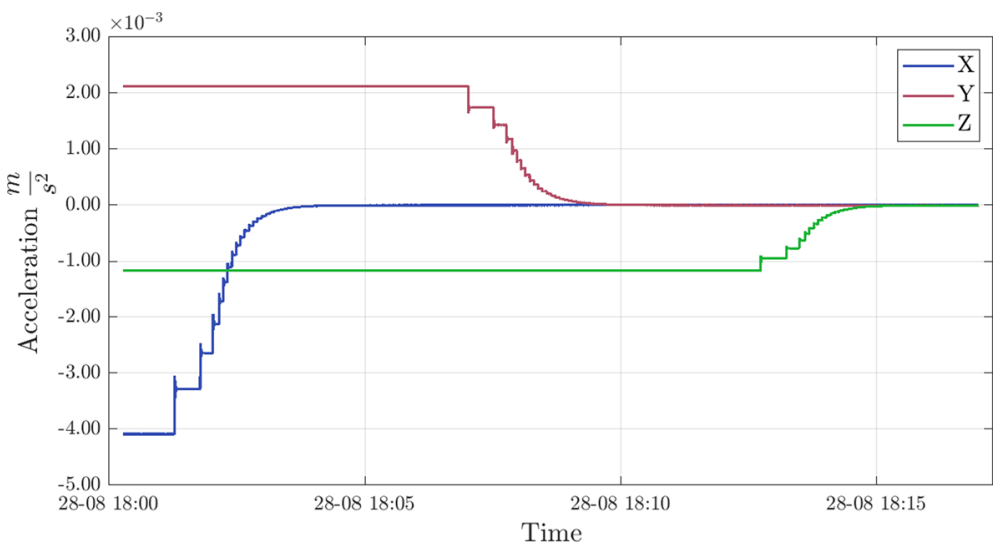

Fig. 15 Equivalent acceleration signal during ISA sensing masses recentering phase

- functional verification of ISA internal actuators (e.g. centering of sensing masses, generation of calibration signal and sensors transfer function control).

The 0- $g$ equilibrium positions have been measured with the actuators switched off, hence not applying any force on the proof masses and acquiring the signals from the sensors in this condition. This is the position assumed by the sensing masses when in free fall in the gravitational field; the measured values are the displacement of the equilibrium position with respect to the ideal central position when the pick-up capacitive bridge is at its equilibrium:

- ISA X proof mass (anti-parallel to MPO Z axis): $-5.5 \mu \mathrm{m}$;

- ISA Y proof mass (parallel to MPO Y axis): $2.1 \mu \mathrm{m}$;

- ISA Z proof mass (parallel to MPO X axis): less than $-0.5 \mu \mathrm{m}$.

These measured values are close to the expected ones, i.e. the ones obtained during on ground characterisation, when an indirect measure of these positions was carried out at IAPS by using a custom procedure and experimental setup (Fiorenza et al. 2016) (see Sect. 5). Although the instrument performance verification is beyond the scope of the commissioning activities, such values point to very low expected values of measurement error due to nonlinearity of the response function and low sensitivity to high-frequency (i.e. above $0.1 \mathrm{~Hz}$ ) micro-vibrations.

Once completed the acquisition of data for the zero- $g$ positions, the actuators were activated. This allowed a recentering of the sensing masses (i.e. bringing them to the pick-up bridge equilibrium position) with an accuracy of $0.01 \mu \mathrm{m}$ (see Fig. 15), and a damping of the mechanical oscillator resonance, reducing its quality factor to a value close to $10 .^{8}$

\subsection{Dynamical Environment Characterisation}

Due to its working principle ISA is highly sensitive to the spacecraft mechanical environment, i.e. the micro-vibrations induced by any on-board mechanism. This means, on one side, that challenging requirements had to be placed on the allowed spacecraft vibration levels, on the other side, that ISA can be effectively used to characterise such an environment

\footnotetext{
${ }^{8}$ The need to lower the quality factor derives from the necessity to withstand the micro-vibrations on-board the spacecraft without exceeding the maximum oscillation amplitude of the sensing masses.
} 


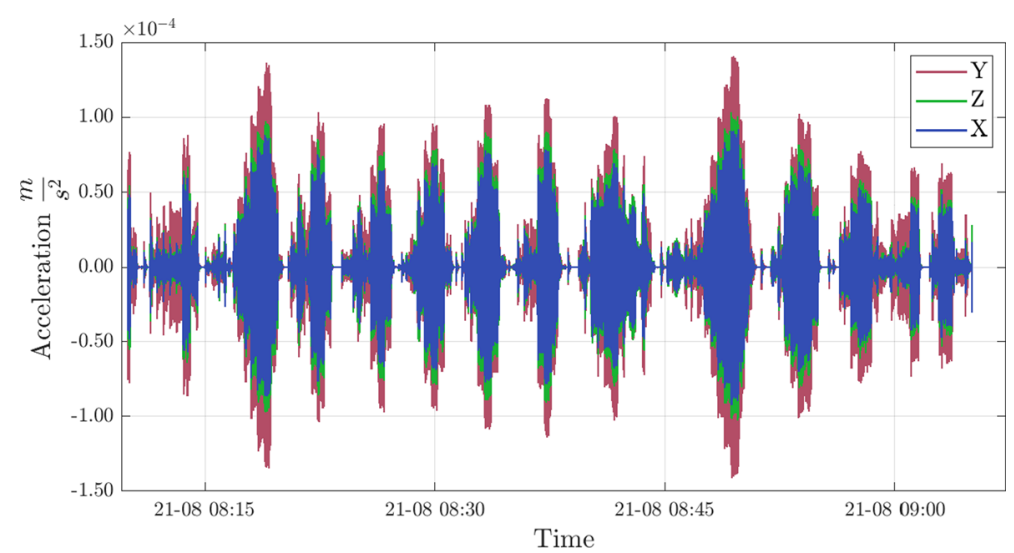

Fig. 16 ISA X, Y, Z axis acquisition with HGA in Earth Tracking mode and MCS in quasi-inertial attitude

and provide relevant information on spacecraft sub-systems. During the NECP, several different mechanical environment conditions have been recorded. In the following vibrations measured in some typical conditions are reported. By correlating ISA output to the TM and operations of other spacecraft systems, it has been possible to identify some sources of these vibrations.

A first identified source of micro-vibrations is the pointing mechanism of the MPO High Gain Antenna (HGA). Depending on the mode such a mechanism is operated, different acceleration spectra and patterns have been observed. When the HGA pointing mechanism is in Earth Tracking mode, a high frequency vibration is well visible in the instrument data (see Fig. 16).

Such a behaviour, with a high-frequency noise modulated with a pattern on time scales of hundreds of seconds, has been recorded only in the above-described combination of HGA pointing mechanism mode and MCS attitude motion. Indeed, in this conditions the HGA pointing mechanism operates with very slow angle corrections (see Fig. 17) to maintain the HGA pointing towards the Earth. In doing this it generates such characteristics vibrations recorded by ISA.

Even though the majority of such signals are out of the ISA scientific band, their effect on ISA measurements is not negligible, as shown in Fig. 18, that reports low-pass filtered ISA measurements. The current understanding of this effect is that the non-linear part of ISA response causes a down-conversion of the high-frequency and modulated input signal. During the cruise phase, activities to further investigate this phenomenon and to tune instrument to reduce its effect are envisaged. ${ }^{9}$

In order to understand the extent of the dependence of recorded signal on the HGA pointing mechanism operations, the above signals can be compared to those recorded with the MCS in quasi-inertial attitude as well, but with the HGA pointing mechanism blocked in its Hold mode. In Fig. 19 the plot of the signal acquired in the same conditions of Fig. 16, except for the HGA pointing mechanism mode, is reported.

\footnotetext{
${ }^{9}$ To counteract such phenomena, ISA embeds a dedicated software functionality consisting in a non-linearity correction; moreover, by correlating the acceleration signal with tracking measurements (by MORE) and attitude data (by AOCS), it will be possible to discriminate between actual acceleration signals and downconversion artifacts.
} 


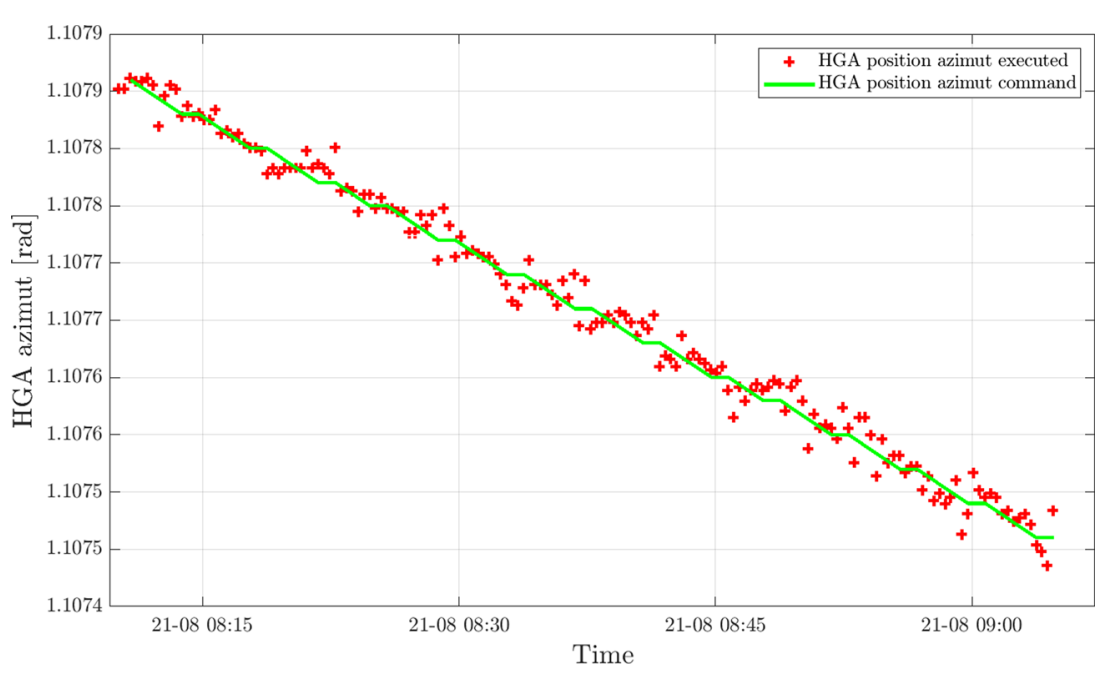

Fig. 17 Azimuth of HGA in Earth Tracking mode, with MCS in quasi-inertial attitude

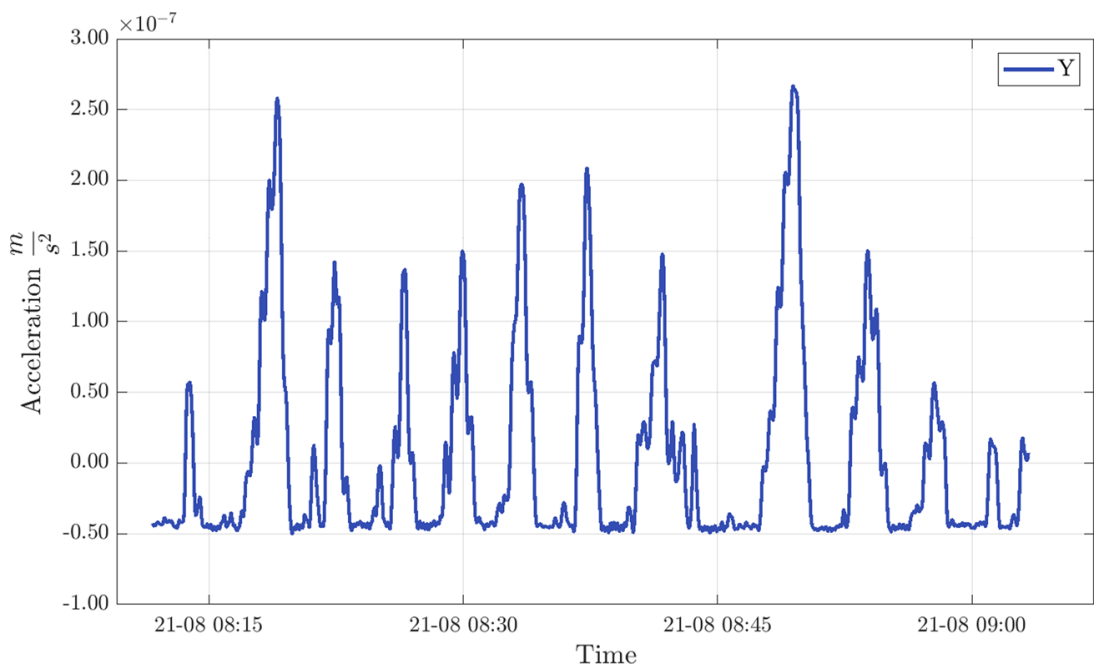

Fig. 18 Low-frequency component of ISA Y channel related to the HGA azimuth corrections while in Earth tracking mode

Figures 20, 21 and 22 report the acquired signal spectra for the three ISA sensors during a low-noise time period recorded on 20 August 2019, from 01:00 to 01:30. For the frequency range $10^{-3} \mathrm{~Hz}-10^{-1} \mathrm{~Hz}$, that is the upper part of ISA scientific measurement band, the level shown can be regarded as the sum of the actual mechanical noise plus the instrumental noise floor. Even though the purpose of the commissioning phase was not the measurement performance verification, a very preliminary estimate can be inferred. The nominal scientific phase is at Mercury and consequently the in-cruise environmental conditions are very different with respect to the design (MCS configuration, larger solar panels, and so on). Nevertheless, the data gathered when the pointing mechanism of HGA is in Hold mode and 


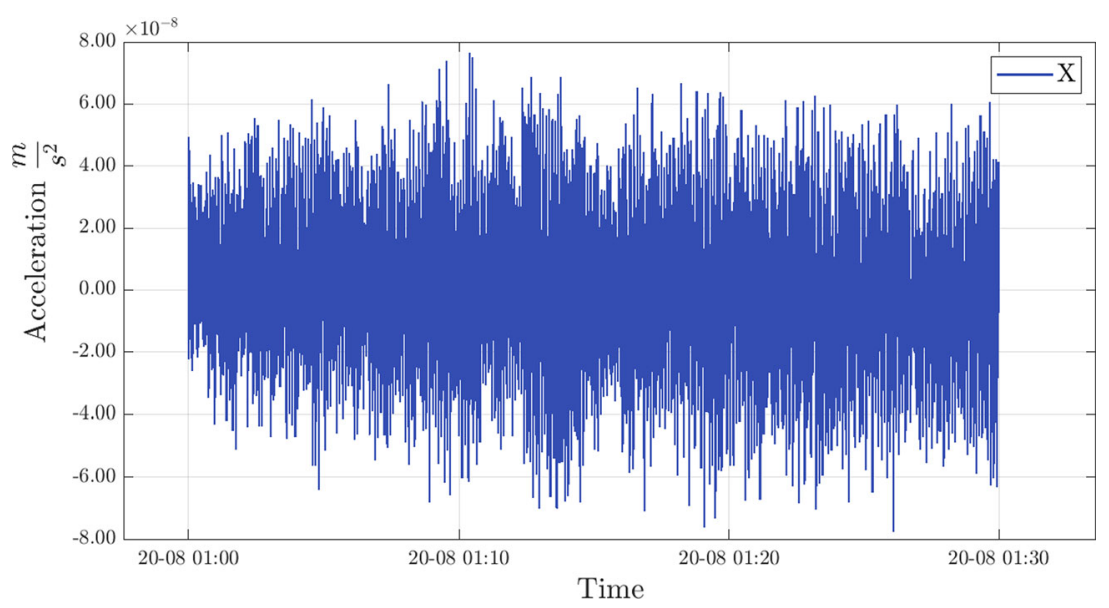

Fig. 19 ISA X axis acquisition with HGA in Hold mode and MCS in quasi-inertial attitude (20 August 2019, from 01:00 to 01:30)

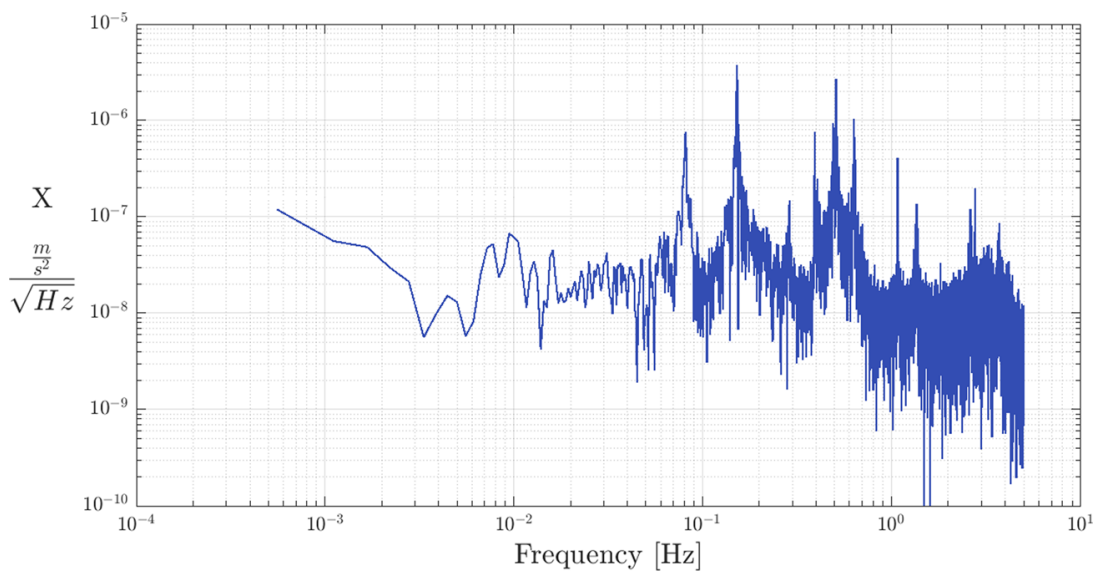

Fig. 20 PSD of acceleration for ISA X axis during a low-noise time period (20 August 2019, from 01:00 to 01:30)

MCS in quasi-inertial attitude can be used to define an upper bound in the noise floor in such a configuration. In the lower part of the scientific band, $3 \times 10^{-5} \mathrm{~Hz}-10^{-3} \mathrm{~Hz}$, the level is affected by thermal effects; indeed, at the time of those acquisitions, the tuning of ISA thermal control system to cruise conditions was not yet completed.

A second identified source of micro-vibrations is given by the reaction wheels. Indeed, acceleration peaks are present in ISA measurements, whose frequencies are directly related to the wheels rotation speeds. This is particularly apparent during slew phases (see Fig. 14). It has to be noticed that even though such a signal is well visible in ISA measurements, it is out of the scientific frequency band and does not cause any harm to the instrument operations or performance.

As expected, another clearly visible signal in ISA measurements during the cruise phase is caused by variations in the rotation speed of the spacecraft. Figure 23 and Fig. 24 re- 


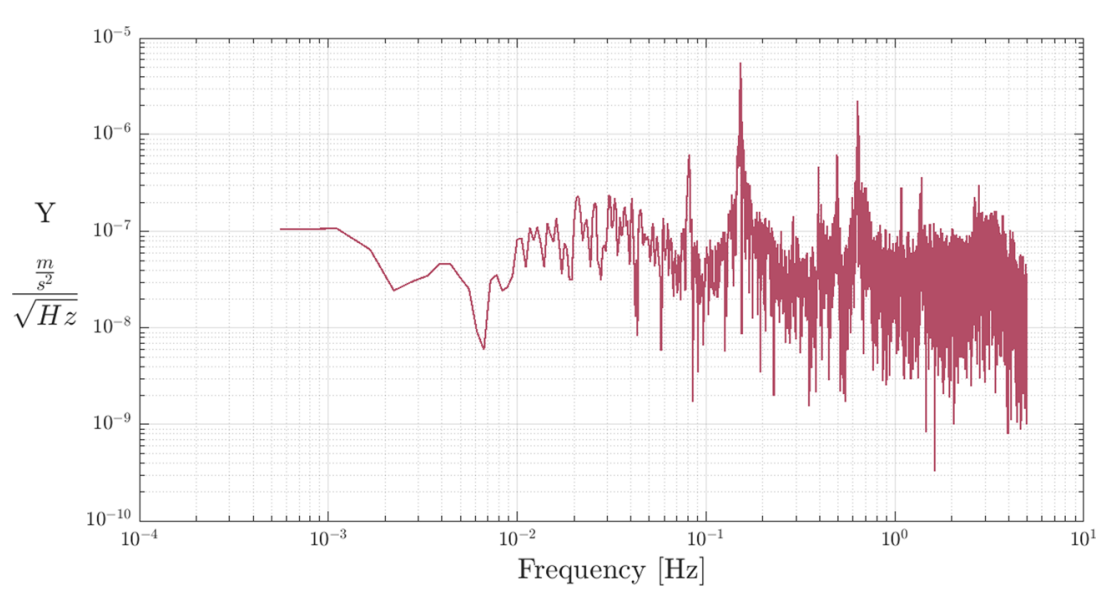

Fig. 21 PSD of acceleration for ISA Y axis during a low-noise time period (20 August 2019, from 01:00 to 01:30)

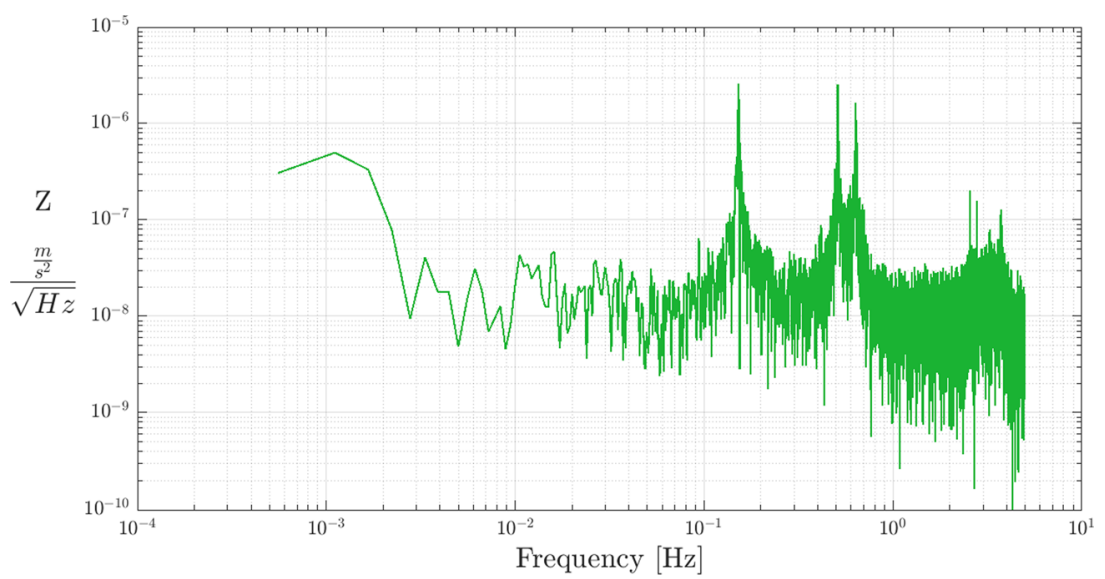

Fig. 22 PSD of acceleration for ISA $Z$ axis during a low-noise time period (20 August 2019, from 01:00 to 01:30)

port respectively the MCS angular rate profiles during a manoeuvre and the accelerations measured by ISA during the same period (16 December 2019, from 09:00:00 to 09:16:00).

\section{Conclusions}

The ISA accelerometer has been designed and built, based on pioneering work aimed at developing resonant gravitational wave detectors, to perform high-precision measurements of small acceleration signals, like the ones that show themselves in satellite dynamics due to non-gravitational effects. As such, it was a good candidate to be hosted on space missions devoted (also) to fundamental physics and planetary geodesy research. Indeed, it was selected by European Space Agency as part of a suite of instruments devoted to perform dedicated measurements to investigate Mercury geophysics, through the study of the planet 


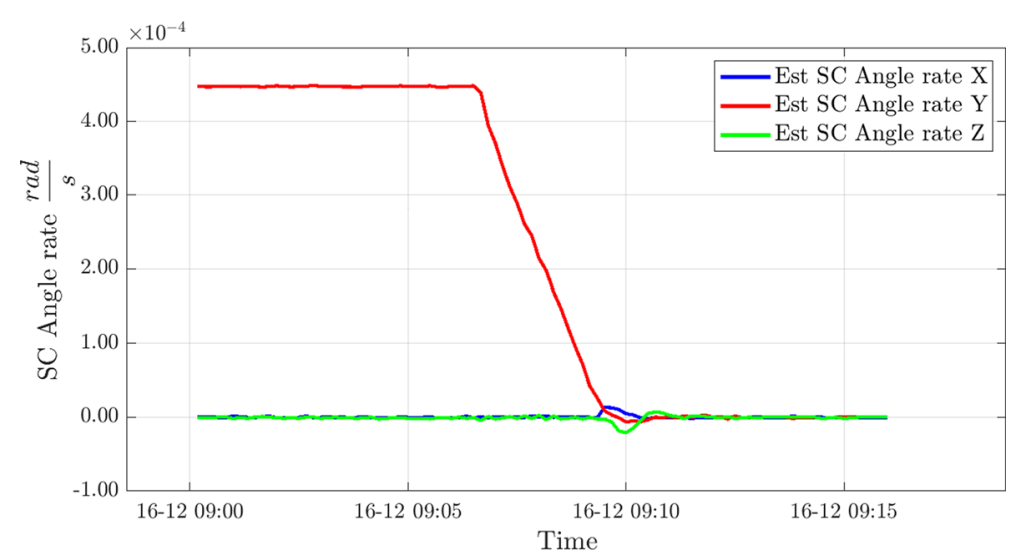

Fig. 23 Estimated spacecraft angle rate by AOCS

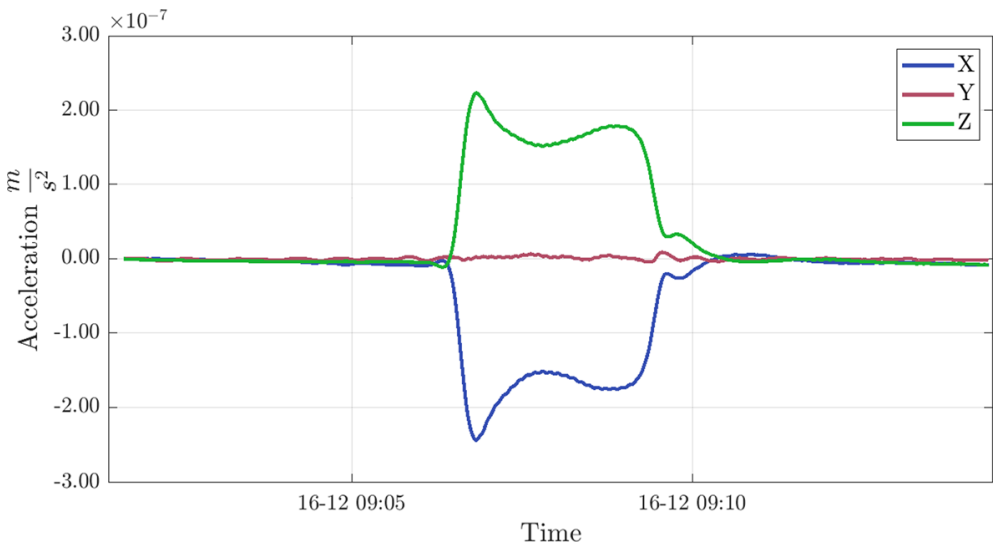

Fig. 24 ISA signals collected during the spacecraft angle rate variation shown in Fig. 23

gravitational field and rotation, and to test the machinery of gravitation relatively close to the Sun. The instrument characteristics have been tuned to cope with the demanding MORE scientific objectives; along with the instrument development, specific concepts, procedures and tools have been devised in order to fully exploit the instrument scientific capabilities.

The first commissioning, performed during the initial cruise phase, confirmed that ISA survived the launch safely and in good health; all its parameters were nominal and, with the tuning of its systems that will be performed during the remaining cruise phase, ISA will be able to contribute to BepiColombo and MORE scientific goals. Indeed, although the instrumental performance verification will be carried out during the Mercury Commissioning Phase (just after Mercury orbit insertion), the data acquired during NECP let us to be confident that the expected performance can be achieved.

During the on ground development, the instrument was subjected to three calibration procedures with the aim of characterising and optimising its operativity and performance within the MORE scope. The calibration of actuators, performed in June-July 2015 by IAPS ISA Science Team, supported by Thales Alenia Space and INRIM, was aimed at establishing a relationship between voltages applied to the plates and corresponding forces applied 
to the sensing mass. The measurements allowed to determine the calibration curve with an accuracy of $600 \mathrm{ppm}$. The calibration of sensing axes alignments was performed at IAPS in 2015 by using a procedure and a set-up ad-hoc developed. The measurements allowed to determine the sensing axes direction with respect to a ISA-fixed reference frame with an accuracy within $20^{\prime \prime}-30^{\prime \prime}$ per axis $(2 \sigma)$. At last, although established on ground, the calibration of the transduction factor, a proportionality term between the acceleration and the electrical output provided by each accelerometer, is a measurement that will be repeated in orbit, since its value depends on other sensor parameters and on the environmental conditions.

The commissioning activities that have been performed till now, besides testing the instrument functionality, proved to be a valid test for the on ground system dedicated to ISA data handling and archiving that is being developed. Such a system is almost complete, and has been proved to be ready to support further cruise science and commissioning activities. As a by-product of the commissioning activities, ISA produced interesting data useful to characterise the spacecraft mechanical environment. Indeed, in this period the external perturbations acting on the spacecraft were almost negligible, so that the acquired accelerations are mainly those related to the on-board mechanical environment. Even though the spacecraft configuration is different from the one during Mercury Science Phase (indeed, during cruise the spacecraft is composed by the two orbiters of the mission and a cruise module), the acquired data give useful information for the planning of the commissioning and science activities to be performed once orbiting around Mercury.

Finally, it has to be highlighted once more that ISA is the first high-sensitivity accelerometer to fly on-board an interplanetary mission and as such, a likely source of interesting discoveries.

Acknowledgements The present work has been done in cooperation and with the support of Italian Space Agency - ASI (under cooperation agreement n. 2017-47-H.0) and European Space Agency - ESA (under contract No. 4000119356/16/ES/JD).

Funding Note Open access funding provided by Istituto Nazionale di Astrofisica within the CRUI-CARE Agreement.

Publisher's Note Springer Nature remains neutral with regard to jurisdictional claims in published maps and institutional affiliations.

Open Access This article is licensed under a Creative Commons Attribution 4.0 International License, which permits use, sharing, adaptation, distribution and reproduction in any medium or format, as long as you give appropriate credit to the original author(s) and the source, provide a link to the Creative Commons licence, and indicate if changes were made. The images or other third party material in this article are included in the article's Creative Commons licence, unless indicated otherwise in a credit line to the material. If material is not included in the article's Creative Commons licence and your intended use is not permitted by statutory regulation or exceeds the permitted use, you will need to obtain permission directly from the copyright holder. To view a copy of this licence, visit http://creativecommons.org/licenses/by/4.0/.

\section{References}

P. Cappuccio, A.D. Ruscio, L. Iess, M.J. Mariani, BepiColombo Gravity and Rotation Experiment in a Pseudo Drag-Free System (2020). https://doi.org/10.2514/6.2020-1095

M. Costa, SPICE for ESA planetary missions, in Planetary Science Informatics and Data Analytics Conference, vol. 2082 (2018), p. 6008

M. Drinkwater, R. Haagmans, D. Muzi, A. Popescu, R. Floberghagen, M. Kern, M. Fehringer, The Goce gravity mission: Esa's first core Earth explorer, in Proceedings of the 3rd International GOCE User Workshop, 6-8 November, 2006, Frascati, Italy. ESA Special Publication, SP-627 (2007), pp. 1-8 
ESA, Planetary Science Archive. Tech. Rep. (2020a). https://archives.esac.esa.int/psa

ESA, SCOS-2000. Tech. Rep. (2020b). https://www.esa.int/Enabling_Support/Operations/gse/SCOS-2000

E. Fiorenza, M. Lucente, C. Lefevre, F. Santoli, V. Iafolla, Zero-g positioning for the BepiColombo ISA accelerometer. Sens. Actuators A, Phys. 240, 31-40 (2016). https://doi.org/10.1016/j.sna.2016.01.036. http://www.sciencedirect.com/science/article/pii/S092442471630036X

J. Flury, S. Bettadpur, B.D. Tapley, Precise accelerometry onboard the GRACE gravity field satellite mission. Adv. Space Res. 42(8), 1414-1423 (2008). https://doi.org/10.1016/j.asr.2008.05.004

A. Genova, T. Hauke Van Hoolst, D. Heyner, L. Iess, F. Santoli, K. Thomas, M. Langlais, S. Oliveira, W. Tosi, J. Benkhoff, Geodesy, geophysics and fundamental physics investigations of the BepiColombo mission. Space Sci. Rev. (2020, this issue)

C.E. Griggs et al., Levitated Superconducting Gravity Gradiometer for Planetary Missions (2014)

V. Iafolla, S. Nozzoli, Italian spring accelerometer (ISA) a high sensitive accelerometer for "BepiColombo" ESA CORNERSTONE. Planet. Space Sci. 49(14-15), 1609-1617 (2001). https://doi.org/10.1016/ S0032-0633(01)00097-6

V. Iafolla, S. Nozzoli, E. Fiorenza, One axis gravity gradiometer for the measurement of Newton's gravitational constant G. Phys. Lett. A 318(3), 223-233 (2003). https://doi.org/10.1016/j.physleta.2003.07.014

V. Iafolla, D.M. Lucchesi, S. Nozzoli, F. Santoli, ISA accelerometer onboard the Mercury Planetary Orbiter: error budget. Celest. Mech. Dyn. Astron. 97(3), 165-187 (2007). https://doi.org/10.1007/s10569-0069059-0

V. Iafolla, E. Fiorenza, C. Lefevre, A. Morbidini, S. Nozzoli, R. Peron, M. Persichini, A. Reale, F. Santoli, Italian spring accelerometer (ISA): a fundamental support to BepiColombo radio science experiments. Planet. Space Sci. 58(1-2), 300-308 (2010). https://doi.org/10.1016/j.pss.2009.04.005

V. Iafolla, E. Fiorenza, C. Lefevre, D.M. Lucchesi, M. Lucente, C. Magnafico, R. Peron, F. Santoli, The BepiColombo ISA accelerometer: ready for launch, in 2016 IEEE Metrology for Aerospace (MetroAeroSpace) (2016), pp. 538-544. https://doi.org/10.1109/MetroAeroSpace.2016.7573273

L. Iess et al., Gravity, geodesy and fundamental physics with BepiColombo's MORE investigation. Space Sci. Rev. (2020, this issue)

L. Imperi, L. Iess, Testing general relativity during the cruise phase of the BepiColombo mission to Mercury, in 2015 IEEE Metrology for Aerospace (MetroAeroSpace) (2015), pp. 135-140. https://doi.org/10.1109/ MetroAeroSpace.2015.7180641

L. Imperi, L. Iess, M.J. Mariani, An analysis of the geodesy and relativity experiments of BepiColombo. Icarus 301, 9025 (2018). https://doi.org/10.1016/j.icarus.2017.09.008

JPL-NASA, SPICE. An observation geometry system for space science missions. Tech. Rep. (2020). https:// naif.jpl.nasa.gov/naif

R.P. Kornfeld, B.W. Arnold, M.A. Gross, N.T. Dahya, W.M. Klipstein, P.F. Gath, S. Bettadpur, GRACE-FO: the gravity recovery and climate experiment follow-on mission. J. Spacecr. Rockets 56(3), 931-951 (2019). https://doi.org/10.2514/1.A34326

D. Koschny, J. Benkhoff, H. Laakso, J. Mc Auliffe, H. Middleton, S. de la Fuente, J. Lefort, The European BepiColombo science ground segment—overview and tasks. Planet. Space Sci. 58(1-2), 319-326 (2010). https://doi.org/10.1016/j.pss.2009.07.006

D.M. Lucchesi, V. Iafolla, The non-gravitational perturbations impact on the BepiColombo radio science experiment and the key rôle of the ISA accelerometer: direct solar radiation and albedo effects. Celest. Mech. Dyn. Astron. 96, 99-127 (2006). https://doi.org/10.1007/s10569-006-9034-9

J.L. Margot, S.A.I. Hauck, E. Mazarico, S. Padovan, S.J. Peale, Mercury's Internal Structure. Cambridge Planetary Science (Cambridge University Press, Cambridge, 2018), pp. 85-113. Chap. 4

A. Milani, G.F. Gronchi, Theory of Orbit Determination (Cambridge University Press, Cambridge, 2010)

A. Milani, A.M. Nobili, P. Farinella, Non-gravitational Perturbations and Satellite Geodesy (Hilger, Bristol, 1987)

A. Milani, A. Rossi, D. Vokrouhlický, D. Villani, C. Bonanno, Gravity field and rotation state of Mercury from the BepiColombo radio science experiments. Planet. Space Sci. 49, 1579-1596 (2001)

A. Milani, D. Vokrouhlický, D. Villani, C. Bonanno, A. Rossi, Testing general relativity with the BepiColombo radio science experiment. Phys. Rev. D 66(8), 082,001 (2002)

NASA, Planetary Data System. Tech. Rep. (2020). https://pds.nasa.gov

S.J. Peale, Generalized Cassini's laws. Astron. J. 74, 483 (1969). https://doi.org/10.1086/110825

S.J. Peale, Does Mercury have a molten core? Nature 262(5571), 765-766 (1976). https://doi.org/10.1038/ $262765 \mathrm{a} 0$

F. Perez-Lopez, S. Martinez, I.O. de Landaluce, N. Fajersztejn, M. Freschi, M. Fernandez, M. Casale, The Science Operations Quicklook Analysis System for BepiColombo MPO (2016). https://doi.org/10.2514/ 6.2016-2314 
M. Pisani, M. Astrua, V. Iafolla, F. Santoli, D. Lucchesi, C. Lefevre, M. Lucente, On-ground actuator calibration for ISA - BepiColombo, in 2015 IEEE Metrology for Aerospace (MetroAeroSpace) (2015), pp. 312-317. https://doi.org/10.1109/MetroAeroSpace.2015.7180674

M. Pisani, M. Astrua, V. Iafolla, F. Santoli, D. Lucchesi, C. Lefevre, M. Lucente, Characterization of ISA - BepiColombo internal calibration source, in 2016 IEEE Metrology for Aerospace (MetroAeroSpace) (2016), pp. 495-499. https://doi.org/10.1109/MetroAeroSpace.2016.7573265

G. Pizzella, Birth and initial developments of experiments with resonant detectors searching for gravitational waves. Eur. Phys. J. H 41(4), 267-302 (2016). https://doi.org/10.1140/epjh/e2016-70036-8

C. Reigber, H. Lühr, L. Grunwaldt, C. Förste, R. König, H. Massmann, C. Falck, CHAMP Mission 5 Years in Orbit (2006), p. 249. https://doi.org/10.1007/3-540-29522-4_1

G. Schettino, S. Cicalò, S. Di Ruzza, G. Tommei, The relativity experiment of MORE: global full-cycle simulation and results, in 2015 IEEE Metrology for Aerospace (MetroAeroSpace) (2015), pp. 141-145. https://doi.org/10.1109/MetroAeroSpace.2015.7180642

G. Schettino, L. Imperi, L. Iess, G. Tommei, Sensitivity study of systematic errors in the BepiColombo relativity experiment, in 2016 IEEE Metrology for Aerospace (MetroAeroSpace) (2016), pp. 533-537. https://doi.org/10.1109/MetroAeroSpace.2016.7573272

G. Schettino, D. Serra, G. Tommei, A. Milani, Addressing some critical aspects of the BepiColombo MORE relativity experiment. Celest. Mech. Dyn. Astron. 130(11), 72 (2018). https://doi.org/10.1007/s10569018-9863-3

C.M. Will, The confrontation between general relativity and experiment. Living Rev. Relativ. 17(1), 4 (2014) 